\title{
Beyond Graph Neural Networks with Lifted Relational Neural Networks
}

\author{
Gustav Sourek * $\quad$ Filip Zelezny $\quad$ Ondrej Kuzelka \\ Department of Computer Science \\ Faculty of Electrical Engineering \\ Czech Technical University in Prague
}

\begin{abstract}
We demonstrate a declarative differentiable programming framework based on the language of Lifted Relational Neural Networks, where small parameterized logic programs are used to encode relational learning scenarios. When presented with relational data, such as various forms of graphs, the program interpreter dynamically unfolds differentiable computational graphs to be used for the program parameter optimization by standard means. Following from the used declarative Datalog abstraction, this results into compact and elegant learning programs, in contrast with the existing procedural approaches operating directly on the computational graph level. We illustrate how this idea can be used for an efficient encoding of a diverse range of existing advanced neural architectures, with a particular focus on Graph Neural Networks (GNNs). Additionally, we show how the contemporary GNN models can be easily extended towards higher relational expressiveness. In the experiments, we demonstrate correctness and computation efficiency through comparison against specialized GNN deep learning frameworks, while shedding some light on the learning performance of existing GNN models.
\end{abstract}

\section{Introduction}

It has been recently proposed by several authors that incorporating logic reasoning capabilities into neural networks is crucial to achieve more powerful AI systems (Marcus, 2020; De Raedt et al., 2020, Lamb et al., 2020). Indeed, we see a rising interest in enriching deep learning models with certain facets of symbolic AI, ranging from logical entailment (Evans et al., 2018), rule learning (Evans and Grefenstette, 2017), and solving combinatorial problems (Palm et al., 2018), to proposing differentiable versions of a whole Turing machine (Graves et al., 2014, 2016). However, similarly to the Turingcompleteness of recurrent neural networks, the expressiveness of these advanced neural architectures is not easily translatable into actual learning performance, as their optimization tends to be often prohibitively difficult (Lipton et al., 2015).

There has been a long stream of research in neural-symbolic integration (Bader and Hitzler, 2005 Garcez et al. 2019), traditionally focused on emulating logic reasoning within neural networks (Towell et al. 1990; Smolensky, 1990; Botta et al., 1997; Ding and Liya Ding, 1995). The efforts eventually evolved from propositional (Towell et al., 1990; Garcez and Zaverucha, 1999) into full first order logic settings, mapping logic constructs and semantics into respective tensor spaces and optimization constraints (Serafini and d'Avila Garcez, 2016; Dong et al., 2019, Marra et al., 2020). Traditionally, the

*corresponding author: souregus@fel.cvut.cz 
neural-symbolic works focus on providing various perspectives into the correspondence between symbolic and sub-symbolic representations and computing, targeting again mostly novelty and theoretical expressiveness rather than practical learning applications.

From the bottom-up practical perspective, there has been a continuous effort of applying neural network learning to increasingly complex relational data (Uwents et al., 2011; Dash et al., 2018; Kazemi and Poole, 2018). While learning from relational data has been traditionally dominated by approaches rooted in relational logic (Muggleton and De Raedt, 1994) and its probabilistic extensions (Kersting and De Raedt, 2001; Richardson and Domingos, 2006a; De Raedt et al., 2007), the neural networks offer highly efficient latent representation learning, which is beyond capabilities of the symbolic systems. Neural networks on the other hand have traditionally been based on fixed tensor representations, which cannot explicitly capture the unbounded, dynamic and irregular nature of the relational data.

Recursive (Socher et al. , 2013b) and Graph neural networks (GNNs) (Scarselli et al., 2008) introduced a highly successful paradigm shift by moving from fixed neural architectures to dynamically constructed computation graphs, directly following the structural bias presented by the input examples. As opposed to the recurrent (Graves et al., 2014) and "tensorization" (Garcez et al., 2019) neural-symbolic approaches, this enables to exploit the structural properties of the data more efficiently, as they are simply directly coded into the very structure of the model, similarly to the original propositional neural-symbolic integration methods (Towell et al., 1990). Consequently, GNNS achieved remarkable success in a wide range of tasks (Zhou et al., 2018).

In targeting integration of deep and relational learning, one of the core desired properties for an integrated system is to keep expressiveness of both the worlds as a special case. While much focus has been devoted to keep the expressiveness of the logic reasoning, considerably less attention was put on the neural models, the expressiveness and modern variations of which are mostly ignored by the integrated systems (Manhaeve et al., 2018).

In this paper, we show how to use simple relational logic programs to capture advanced convolutional neural architectures in a tightly integrated and exact manner. Particularly, we use the language of Lifted Relational Neural Networks (LRNNs) (Sourek et al., 2018), and demonstrate that a wide range of neural models, ranging from simple perceptrons to complex GNNs, can be elegantly and efficiently covered - not only from the perspective of expressiveness but, importantly, from the practical point of view 1 . We further show how to easily extend the basic GNN idea into some of the most contemporary GNN architectures, and beyond.

The paper is structured as follows. Firstly, we introduce the necessary preliminaries of logic and deep learning in Section 2. In Section3, we introduce the language of LRNNs, which we use throughout the paper. Subsequently, we illustrate LRNNs on a range of example models in Section 4. Capturing and extending GNNs is then detailed in Section 5. In Section 6, we demonstrate practicality and efficiency of the approach. We then discuss related works in Section 7 and conclude in Section 8 .

\section{Background}

Here we introduce the necessary preliminaries of (i) logic programming and (ii) deep learning, which we seek to integrate.

\section{$2.1 \quad$ Logic}

Syntax: A relational logic theory is a set of formulas formed from constants, variables, and predicates (Smullyan, 1995). Constant symbols represent objects in the domain of interest (e.g. hydrogen $)_{1}$ and

\footnotetext{
${ }^{1}$ Code to reproduce experiments from this paper is available at https://github.com/GustikS/GNNwLRNNs The LRNNs framework itself can then be found at https://github.com/GustikS/NeuraLogic
} 
will be written in lower-case. Variables (e.g. $X$ ) range over the objects in the domain and will be written with a capitalized first letter. Predicate symbols represent relations among objects in the domain or their attributes. A term may be a constant or variable. An atom is a predicate symbol applied to a tuple of terms (e.g. bond $\left(X\right.$, hydrogen $\left.\left._{1}\right)\right)$. Formulas are constructed from atoms using logical connectives of $\vee$ and $\wedge$ (Smullyan, 1995). A ground term is a term containing no variables. A ground atom, also called proposition, is an atom having only ground terms as arguments (e.g. bond( oxygen $_{1}$, hydrogen 1$)$ ). A literal is an atom or a negation of an atom. A clause is a universally quantified disjunction of literals 2 , A clause with exactly one positive literal is a definite clause. A definite clause with no negative literals (i.e. consisting of just one literal) is called a fact. A definite clause $h \vee \neg b_{1} \vee \cdots \vee \neg b_{k}$ can also be written as an implication $h \leftarrow b_{1} \wedge \cdots \wedge b_{k}$. The literal $h$ is then called head and the conjunction $b_{1} \wedge \cdots \wedge b_{k}$ is called body. We will often call definite clauses, which are not facts, rules. A set of such rules is then commonly called a logic program.

Semantics: The Herbrand base of a set of relational formulas $\mathcal{P}$ is the set of all ground atoms which can be constructed using the constants and predicates that appear in this set. A Herbrand interpretation of $\mathcal{P}$, also called a possible world, is a mapping that assigns a truth value to each element from $\mathcal{P}$ 's Herbrand base. We say that a possible world $I$ satisfies a ground atom $F$, written $I \models F$, if $F \in I$. The satisfaction relation is then generalized to arbitrary ground formulas in the usual way. A set of ground formulas is satisfiable if there exists at least one possible world in which all formulas from the set are true; such a possible world is called a Herbrand model. Each set of definite clauses has a unique Herbrand model that is minimal w.r.t. the subset relation, called its least Herbrand model. The least Herbrand model of a finite set of ground definite clauses can be constructed in a finite number of steps using the immediate-consequence operator (Van Emden and Kowalski, 1976). This immediate consequence operator is a mapping $T_{p}$ from Herbrand interpretations to Herbrand interpretations, defined for a set of ground definite clauses $\mathcal{P}$ as $T_{p}(I)=\left\{h \mid\left(h \leftarrow b_{1} \wedge \cdots \wedge b_{k}\right) \in \mathcal{P},\left\{b_{1}, \ldots, b_{k}\right\} \subseteq I\right\}$. In other words, the operator $T_{p}$ expands the current set of true atoms (i.e. the current Herbrand interpretation $I$ ) with their immediate consequences as prescribed by the rules in $\mathcal{P}$.

Now consider a set of non-ground definite clauses $\mathcal{P}$. The grounding of a clause $\alpha$ from $\mathcal{P}$ is the set of ground clauses $G(\alpha)=\left\{\alpha \theta_{1}, \ldots, \alpha \theta_{n}\right\}$ where $\theta_{1}, \ldots, \theta_{n}$ is the set of all possible substitutions, each mapping the variables occurring in $\alpha$ to constants appearing in $\mathcal{P}$. Note that if $\alpha$ is already ground, its grounding is a singleton. The grounding of $\mathcal{P}$ is given by $G(\mathcal{P})=\bigcup_{\alpha \in \mathcal{P}} G(\alpha)$. The least Herbrand model of $\mathcal{P}$ is then defined as the least Herbrand model of $G(\mathcal{P})$. In practice, most of the rules in the grounding $G(\mathcal{P})$ will be irrelevant, as their body can never be satisfied. The restricted grounding limits the grounding to those rules which are "active", i.e. whose body is satisfied in the least Herbrand model $\mathcal{H}$. It is defined by $G^{R}(\mathcal{P})=\left\{h \theta \leftarrow b_{1} \theta \wedge \cdots \wedge b_{k} \theta \mid\left(h \leftarrow b_{1} \wedge \cdots \wedge b_{k}\right) \in \mathcal{P}\right.$ and $\left.\left\{h \theta, b_{1} \theta, \ldots, b_{k} \theta\right\} \subseteq \mathcal{H}\right\}$.

\subsubsection{Logic Programming}

Logic programming is a declarative programming paradigm for computation with logic programs. In this paradigm, definite clauses are used to express facts and rules about a domain, and the computation is then carried out by the means of logical inference. Syntactically, the rules in the program $h \leftarrow$ $b_{1} \wedge \cdots \wedge b_{k}$ are commonly written as

$$
1 \mathrm{~h}:-\mathrm{b}_{1}, \ldots, \mathrm{b}_{k} \text {. }
$$

where each ", " stands for conjunction, and ":-" replaces the implication, which reads right-to-left. Facts are then simply rules with no body.

\footnotetext{
${ }^{2}$ Note we do not write the universal quantifiers explicitly in this paper.
} 
Particularly, we consider the language of Datalog (Unman, 1989), a restricted function-free subset of Prolog (Bratko, 2001). Datalog is a domain specific language used in advanced deductive database engines. In contrast with Prolog, Datalog is a truly declarative language, where the order of clauses does not influence execution, and it is also guaranteed to terminate. Separate efficient theorem proving engine can then be used for computing the execution inferences (Bancilhon et al. 1985).

Importantly, it is still a relational language, and one can thus use variables in the clauses, enabling to compose general and reusable programming patterns, such as

$$
1 \mathrm{~h}(\mathrm{X}) \text { :- } \operatorname{edge}(\mathrm{X}, \mathrm{Y}) \text {, node }(\mathrm{Y}) \text {. }
$$

which will then be automatically bound to a multitude of ground data structures via different substitutions for the variables $\{\mathrm{X}, \mathrm{Y}\}$. We will further extensively use this non-ground expressiveness while extending Datalog towards differentiable programming

Query: Similarly to querying a standard database with SQL, one provides a query atom $(q)$ to execute a Datalog program towards inference of a specific target, e.g.

$$
1 \mathrm{~h}\left(\text { oxygen }_{1}\right) \text { ? }
$$

which then drives the theorem proving engine to find a model $I=q$ of the given logic program. If successful, the result of the execution is then the query together with the possible world $I$ used for its derivation. Note that there may be multiple worlds that model $q$.

\section{$2.2 \quad$ Deep Learning}

Deep learning is a machine learning approach characterized by using multi-layered neural network models. A neural network is a parameterized computation graph, particularly a data-flow graph where the data flowing through the edges are being successively transformed by numeric operations represented by the nodes (neurons). A neural layer is a set of neurons residing at the same depth in the directed data-flow graph. A multi-layered ("deep") neural network is a graph with more than two such layers.

The data are commonly represented as (fixed-size) tensors, and the operations are commonly differentiable non-linear functions. Owing to the differentiability of the functions, the parameters of a graph, commonly associated with the edges, can be efficiently trained by gradient-descend routines. Due to the increasingly complex nature of the computation graphs and the utilized operations, the field has been recently also referred to as differentiable programming ${ }^{4}$.

Neural architectures are common design patterns used in creation of the computation graphs for specific types of problems. Here we briefly review some of the most common and successful neural architectures used in deep learning 5

\footnotetext{
${ }^{3}$ This is a distinguishing feature from many other procedural differentiable programming languages, such as PyTorch or TensorFlow, which are effectively propositional in this sense.

${ }^{4}$ Note however that, despite being theoretically Turing-complete (e.g. recurrent neural networks), the models themselves are rarely as expressive in practice as standard programming languages used for their creation.

${ }^{5}$ We introduce these architectures explicitly, despite being commonly known, as we further work with them in detail as differentiable Datalog programs.
} 


\subsubsection{Multi-layer Perceptrons}

A multi-layered perceptron (MLP) is the original and most common neural architecture. It is a directed feed-forward data-flow graph. Moreover, the interconnections between nodes in subsequent layers commonly follow the "fully-connected" pattern (a complete bipartite graph). Consequently, assuming the common vector form of the input data, the computation graph can be efficiently reduced to a linear series of dense matrix multiplications, each followed by an element-wise application of a non-linear function, such as logistic sigmoid $(\sigma)$ or rectified linear unit $(R e L U)$.

The main idea behind MLPs is "representation learning" of the input data, often referred to as embedding, where one can think of outputs of the individual layers as transformed representations of the input, each extracting gradually more expressive information w.r.t. the output learning target.

\subsubsection{Convolutional Networks}

A convolutional neural network $(\mathrm{CNN})$ is also a feed-forward architecture, characterized by utilizing particular operations in one or more sub-parts of the computational graph. The specific operations are commonly referred to as "convolution" (filtering) and "pooling". Given a vector input of size $n$, the convolutional filter (kernel) will also be represented by a vector of size $k<n$, which is then successively element-wise multiplied with all the $k$-length subsequences of the input vector, to produce $n-k+1$ scalar values. The resulting values are commonly referred to as "feature-maps". The second operation is the pooling, which aggregates values from predefined spatial sub-regions of the input values (featuremaps) into a single output through application of some (non-parameterized) aggregation function such as mean $(a v g)$ or maximum $(\max )$. The layers of these operations can then be mixed together with the previously introduced layers from MLPs in various combinations.

The main idea behind the convolution operation is the application of the very same parameterized function over different regions of the input. This enables to abstract away common patterns out of different sub-parts of the input representation. The main idea behind the pooling operation is to enforce invariance w.r.t. translation of the inputs.

\subsubsection{Recursive and Recurrent Networks}

A Recursive Neural Network $(\mathrm{RNN})^{6}$ is a neural architecture which differs significantly from the previous in that the exact form of the computation graph is not given in advance. Instead, the computation graph structure directly follows the structure of an input example, which takes the form of a $k$-regular tree. This enables to learn neural networks directly from differently-structured regular tree examples, as opposed to the fixed-size tensors which can be seen as graphs with completely regular grid topologies.

The leaf nodes in the computation tree then represent the input data, each of which is associated with a feature vector (embedding). Every $k$ leaf nodes are consequently combined by a given operation to compute the representation for their common parent node. This combining operation then continues recursively for all interior nodes, until the representation for the root node is computed, which forms the output of the model. Similarly to the convolution in CNNs, the parameterized combining operation over the children nodes remains the same over the whole tree (Socher et al. 2013a) 7 .

The main idea behind recursive networks is that neural learning can be extended towards structured data by generating a dynamic computation graph for each individual example tree. The learning

\footnotetext{
${ }^{6}$ Note that the abbreviation is also used for the recurrent neural networks, in this paper however, we use it solely to refer to recursive networks.

${ }^{7}$ In some works, this architecture is further extended to use a set of different parameterizations, depending for instance on given types associated with the nodes, such as types of constituents in constituency-based parse trees.
} 
then exploits the convolution principle to discover the underlying compositionality of the learning representations in recursive structures.

The basic form of a commonly known Recurrent Neural Network (Lipton et al., 2015) can then be seen as a "restriction" of the idea to sequential structures, i.e. linear chains of input nodes 8 The computation graph is then successively unfolded along the input sequence to compute the hidden representation for each node based on the previous node's representation and the current node features (current input). The main idea behind recurrent networks is that the hidden representation can store a sort of state of the computation.

\subsection{Graph Neural Networks}

Graph Neural Networks (GNN) $)^{9}$ can be seen as a further extension of the principle to completely irregular graph structures (Bronstein et al., 2017). Similarly to the recursive networks, they dynamically unfold the computational graph from the input structure for the purpose. However, GNN is a multi-layered feed-forward neural architecture, where the structure of each layer $i$ exactly follows the structure of the whole input graph. Every node $v$ in the graph can now be associated with a feature vector (embedding), forming the input layer $h(v)^{(0)}=$ features $(v)$. Interestingly, however, this is not necessary in general, as the variance in the graph topologies of the individual examples can already provide enough discriminative information on its own. For computation of the next layer $i+1$ representations of the nodes, each node in the graph updates its own representation by aggregating representation vectors of the adjacent nodes ("message passing") via some parameterized update operation. GNNs again exploit the convolution idea, while the same operation is again applied uniformly over the whole graph. Note that in contrast to recursive networks, a different parameterization is typically used at each layer.

The computation at each layer $i$ can be possibly divided into two steps (Xu et al., 2018a), where we firstly aggregate the hidden representations $h(u)$ of the node's $v$ neighbors $u \in \mathcal{N}(v)$ to obtain some activation value as

$$
\operatorname{act}^{(i)}(v)=\text { aggregate }^{(i)}\left(\left\{h(u)^{(i-1)}: u \in \mathcal{N}(v)\right\}\right)
$$

and then we combine this activation value with the node's $v$ own representation $h(v)$, to obtain its new updated representation to be used in the next layer as

$$
h^{(i)}(v)=\text { combine }^{(i)}\left(h(v)^{(i-1)}, \operatorname{act}^{(i)}(v)\right)
$$

This general principle covers a wide variety of the proposed GNN models, which then reduces to the choice of particular "aggregate and combine" operations. For instance in GraphSAGE (Hamilton et al., 2017), the operations are

$$
\operatorname{act}^{(i)}(v)=\max \left\{\operatorname{ReLU}\left(W \cdot h^{(i-1)}(u)\right) \mid u \in \mathcal{N}(v)\right\}
$$

and

$$
h^{(i)}(v)=W_{f} \cdot\left[\left(h(v)^{(i-1)}, a c t^{(i)}(v)\right]\right.
$$

while in the popular Graph Convolutional Networks (Kipf and Welling, 2016), these can be even merged into a single step as

$$
h^{(i)}(v)=\operatorname{ReLU}\left(W \cdot \operatorname{avg}\left\{h^{(i-1)}(u) \mid u \in \mathcal{N}(v) \cup\{v\}\right\}\right)
$$

\footnotetext{
${ }^{8}$ We note that modern recurrent architectures use additional computation constructs to store the hidden state, such as the popular LSTM cells, which are more complex and do not directly follow from the input structure.

${ }^{9}$ recently more popular in the form of "Graph Convolutional Networks", which slightly differ from the original GNN proposal (Scarselli et al. 2008), but share the general principles discussed.
} 
and the same generic principle applies to many other GNN works ( $\mathrm{Xu}$ et al., 2018b; Gilmer et al. 2017; Xu et al. 2018a).

GNNs can be directly utilized for both graph-level as well as node-level classification tasks. For output prediction on the level of individual nodes, we simply apply some activation function on top of its last layer representation query $(v)=\sigma\left(h(v)^{(n)}\right)$. For predictions on the level of the whole graph $\mathcal{G}$, all the node representations need to be aggregated by some pooling operation such as query $(\mathcal{G})=$ $\sigma\left(\operatorname{avg}\left\{h^{(n)}(v) \mid v \in \mathcal{G}\right\}\right)$.

By following the same pattern at each layer $i$, the computation will produce increasingly more aggregated representations, since at layer $i$ each node effectively aggregates representations from its " $i$-hops" neighborhood. Intuitively, the GNN inference can thus be seen as a continuous version of the popular Weisfeiler-Lehman algorithm (Weisfeiler, 2006) for calculating graph fingerprints used for refutation checking in graph isomorphism testing.

A large number of different variants of the original GNNs (Scarselli et al., 2008) have been proposed, recently achieving state-of-the-art empirical performance in many tasks (Wu et al., 2019; Zhou et al., 2018). In essence, each introduced GNN variant came up with a certain combination of common activation and aggregation functions, and/or proposed extending the architecture with additional connections (Xu et al., 2018b) or layers borrowed from other neural architectures (Veličković et al., 2017; Li et al., 2015), nevertheless they all share the same introduced idea of successive aggregation of node representations. For a general overview, we refer to (Wu et al. 2020, Zhou et al. 2018).

Spectral GNNs: Here we discussed "spatially" represented graphs and operations. However, some GNN approaches represent the graphs and the convolution operation in spectral, Fourier-domain $(\overline{\mathrm{Wu}}$ et al. 2020). There the update operation is typically conveyed in the matrix form as

$$
H^{(i)}=f\left(\hat{A} \times H^{(i-1)} \times W_{i-1}\right)
$$

where $\hat{A}$ is an altered 10 adjacency matrix of the graph, encoding the respective neighborhoods, $H^{(i)}$ contains the successive hidden node representations at layer $i$, and $W_{i}$ are the learnable parameters at each layer. However we note that, not considering the specific normalizations and approximations used, these again follow the same "aggregate and combine" principles, and can be rewritten accordingly (Xu et al., 2018a). While theoretically substantiated in graph signal processing, spectral GNN models are generally inadvisable as they introduce substantial limitations in terms of efficiency, learning, generality, and flexibility (Wu et al., 2020), and we do not consider them further in this paper.

Knowledge Base Embeddings: Knowledge Base Embeddings (KBEs) are a set of approaches designed for the task of knowledge base completion (KBC) (Kadlec et al., 2017), i.e. predicting existing (missing) edges in large knowledge graphs. Particularly, these methods approach the task through learning of a distributed representation (embedding) for the nodes. In multi-relational graphs, a representation of the edge (relation) can also be added, forming a commonly used triplet representation of (object, relation, subject). To predict the probability of a given edge in the knowledge graph, KBEs then choose one of a plethora of functions designed to combine $e^{11}$ the three embeddings from the underlying triplet (Kadlec et al., 2017).

\footnotetext{
${ }^{10}$ e.g. $\hat{A}=D^{-\frac{1}{2}}(A+I) D^{-\frac{1}{2}}$, where $D$ is the diagonal node-degree matrix and $I$ is an identity matrix, such as in the original Graph Convolutional Networks (Kipf and Welling, 2016).

${ }^{11}$ Note that there is no need for the "aggregate" operation in KBEs.
} 


\section{The Language of Lifted Relational Neural Networks}

In this paper we follow up on the work of Lifted Relational Neural Networks (LRNNs) (Sourek et al., 2015) which have been introduced as a framework for templated modeling of diverse neural architectures oriented to relational data. It can be understood as a differentiable version of simple Datalog programming, where the templates, encoding various neuro-relational architectures, take the form of parameterized logic programs. It differs from the commonly used frameworks, such as PyTorch or Tensorflow, in its declarative, relational nature, enabling one to abstract away from the procedural details of the underlying computational graphs even further. We explain principles of this abstraction in the following subsections.

\subsection{Syntax: Weighted Logic Programs}

The syntax of LRNNs is derived directly from the Datalog (Unman, 1989) language (Section 2.1), which we further extend with numerical parameters. Note that this has been exploited in many previous works, where the parameters can signify values associated with facts (Bistarelli et al., 2008) or rules (Eisner and Filardo, 2010). Such extensions are typically designed to integrate standard statistical (or probabilistic (De Raedt et al., 2007)) modelling techniques with the high expressiveness of relational representation and reasoning. In this work we seek to integrate Datalog with deep learning, for which we allow each literal in each clause of the logic program to be associated with a tensor weight. A parameterized program, formed by a multitude of such weighted rules, then declaratively encodes all computations to be performed in a given learning scenario. For clarity of correspondence with standard (neural) learning scenarios, we here further split ${ }^{12}$ the program into unit clauses (facts), constituting the learning examples, and definite clauses (rules), constituting the learning template.

\subsubsection{Learning Examples}

The learning examples contain factual description of a given world. For their representation we use weighted ground facts. A learning example is then a set $E=\left\{\left(V_{1}, e_{1}\right), \ldots,\left(V_{j}, e_{j}\right)\right\}$, where each $V_{i}$ is a real-valued tensor and each $e_{i}$ is a ground fact, i.e. expression of the form

$$
\begin{array}{ll} 
& \mathrm{V}_{1}:: \mathrm{p}_{1}\left(c_{1}^{1}, \ldots, c_{q}^{1}\right) . \\
2 & \ldots \\
{ }_{3} & \mathrm{~V}_{\mathbf{j}}:: \mathrm{p}_{n}\left(c_{1}^{n}, \ldots, c_{r}^{n}\right) .
\end{array}
$$

where $p_{1}, \ldots, p_{n}$ are predicates with corresponding arities $q, \ldots, r$, and $c_{i}^{j}$ are arbitrary constants. Standard logical representation is then a special case where each $\mathbf{V}_{\mathbf{i}}=11$. One can either write $1:$ carbon $\left(c_{1}\right)$ or ommit the weight and write bond $\left(c_{1}, o_{2}\right)$. The values do not have to be binary and can represent a "degree of truth" to which a certain fact holds, such as 0.4::aromatic $\left(c_{1}\right)$. The values are also not necessarily restricted to $(0,1)$, and can thus naturally represent numerical features, such as 6::atomicNumber $\left(c_{1}\right)$ or 2.35::ionEnergy $\left(c_{1}\right.$, level $\left._{2}\right)$. Finally the values are not necessarily restricted to scalars, and can thus have the form of feature vectors (tensors), such as $[1.0,-7, \ldots, 3.14]::$ features $\left(c_{1}\right)$.

Ground facts in examples are also not restricted to unary predicates, and can thus describe not only properties of individual objects, but values of arbitrary relational properties. For exam-

\footnotetext{
${ }^{12}$ Note that this split is not necessary in general, and the template can also contain facts, as well as the learning examples may contain rules, such as in ILP learning scenarios.

${ }^{13}$ Since we consider a close world assumption (CWA) and least Herbrand model, one does not enumerate false facts with zero value.
} 
ple, one can assign feature values to edges in graphs, such as describing a bond between two atoms $[2.7,-1]::$ bond $\left(c_{1}, o_{2}\right)$.

There is no syntactical restriction on how these representations can be mixed together, and one can thus select which parts of the data are better modelled with (sub-symbolic) distributed numerical representations, and which parts yield themselves to be represented by purely logical means, and move continuously along this dimension as needed.

Query: Queries $(q)$ (Sec. 2.1.1) represent the classification labels or regression targets associated with an example for supervised learning. They again utilize the same weighted fact representation such as $1:$ :class or $4.7:$ :target $\left(c_{1}\right)$. Note that the target queries again do not have to be unary, and one can thus use the same format for different tasks. For example, for knowledge graph completion, we would use queries such as 1.0::coworker (alice, bob).

\subsubsection{Learning Template}

The weighted logic programs written in LRNNs are then often referred to as templates. Syntactically, a learning template $\mathcal{T}$ is a set of weighted rules $\mathcal{T}=\left\{\alpha_{i},\left\{W_{j}^{\alpha_{i}}\right\}\right\}=\left\{\left(W^{i}, c\right) \leftarrow\left(W_{1}^{i}, b_{1}\right), \ldots,\left(W_{k}^{i}, b_{k}\right)\right\}$ where each $\alpha_{i}$ is a definite clause and each $W_{j}$ is some real-valued tensor, i.e. expressions of the form

$$
\begin{array}{lllll}
1 & \mathbf{W}^{1}:: \mathrm{h}_{1}^{1}(\ldots):-\mathbf{W}_{1}^{1}: \mathrm{b}_{1}^{1}(\ldots), \ldots, \mathbf{W}_{\mathbf{j}}^{1}: \mathrm{b}_{i}^{1}(\ldots) \\
2 \mathbf{W}^{2}:: \mathrm{h}_{1}^{2}(\ldots):-\mathbf{W}_{1}^{2}: \mathrm{b}_{1}^{2}(\ldots), \ldots, \mathbf{W}_{\mathrm{k}}^{2}: \mathrm{b}_{j}^{2}(\ldots) \\
{ }_{3} \ldots \\
{ }_{4} \mathbf{W}^{\mathrm{n}}:: \mathrm{h}_{p}^{q}(\ldots):-\mathbf{W}_{1}^{\mathrm{n}}: \mathrm{b}_{1}^{n}(\ldots), \ldots, \mathbf{W}_{1}^{\mathrm{n}}: \mathrm{b}_{k}^{n}(\ldots) .
\end{array}
$$

where $\mathrm{h}_{i}^{j}$, s and $\mathrm{b}_{i}^{j}$, s are predicates forming positive, not necessarily different, literals, and $\mathrm{W}_{\mathrm{i}}^{\mathrm{j}}$, $\mathrm{s}$ are the associated tensors (also possibly reused in different places).

The template constitutes roughly what neural architecture means in deep learning ${ }^{14}$ - i.e. it does not (necessarily) encode a particular model or knowledge of the problem, but rather a generic mode of computation.

Example 1 Consider a simple template for learning with molecular data, encoding a generic idea that the representation of a (chemical) atom $\left(e . g . h\left(h_{1}\right)\right)$ is dependent on the representation of the atoms adjacent to it. Given that a molecule can be represented by describing the contained atoms (e.g. a $\left(h_{1}\right)$ ) and bonds between them (e.g. $\left.b\left(h_{1}, o_{1}\right)\right)$, we can intuitively encode this idea by a following rule

$$
1 \mathbf{W}_{\mathbf{h}_{1}}:: h(X):-\mathbf{W}_{\mathbf{a}}: a(Y), \mathbf{W}_{\mathbf{b}}: b(X, Y)
$$

Moreover, one might be interested in using the representation of all atoms $(h(X))$ for deducing the representation of the whole molecule, for which we can write

$$
1 \mathbf{W}_{\mathrm{q}}:: q:-\mathbf{W}_{\mathrm{h}_{2}}: h(X) \text {. }
$$

to derive a single ground query atom $(q)$, which can be associated with the learning target of the whole molecule. The concrete semantics of this template then follows in the next section.

\footnotetext{
${ }^{14}$ We deliberately refrain from using the common term of neural "model", since a single template can have multiple logical (and neural) models.
} 
Table 1: Correspondence between the logical ground model and computation graph.

\begin{tabular}{lll} 
Logical construct & Type of node & Notation \\
\hline Ground atom $h$ & Atom node & $A_{h}$ \\
Ground fact $h$ & Fact node & $F_{(h, \vec{w})}$ \\
Ground rule's $\alpha \theta$ body & Rule node & $R_{\left(W_{0}^{c} c \theta \leftarrow W_{1}^{\alpha} b_{1} \theta \wedge \cdots \wedge W_{k}^{\alpha} b_{k} \theta\right)}^{c \theta}$ \\
Rule's $\alpha$ ground head $h$ & Aggregation node & $\left.G_{\left(W_{0}^{c} c \leftarrow \theta_{i}\right.}^{c} c \leftarrow W_{1}^{\alpha} b_{1} \wedge \cdots \wedge W_{k}^{\alpha} b_{k}\right)$
\end{tabular}

\subsection{Semantics: Computational Graphs Defined by LRNNs}

To explain the correspondence between a relational template $\mathcal{T}$ and a "neural architecture", we now describe the mapping that takes the template and a given example description, consisting of ground facts, and produces a standard neural model. Here, "standard neural model" refers to a specific differentiable computational graph.

First, let $\mathcal{N}_{l}$ be the set of rules and facts obtained from the template and a learning example $\mathcal{N}_{l}=$ $\mathcal{T} \cup E_{l}$ by removing all the tensor weights. For instance, if we had a weighted rule $W:: h:-W_{1}: b_{1}, W_{2}: b_{2}$ , we would obtain $h:-b_{1}, b_{2}$. Then we construct the least Herbrand model $\overline{\mathcal{N}_{l}}$ of $\mathcal{N}_{l}$, which can be done using standard theorem proving techniques. One simple option is to employ a bottom-up grounding strategy by repeated application of the immediate consequence operator (Section 2.1 15 . For consequent neural learning, the target query atom $q$ associated with $E_{l}$ must be logically entailed by $\mathcal{N}_{l}$, i.e. present in $\overline{\mathcal{N}}[16$.

Having the least Herbrand model $\overline{\mathcal{N}}_{l}$ containing $q$, we can directly construct a neural computational graph $G_{l}$. Intuitively, the structure of the graph contains all the logical derivations of the target query literal $q$ from the example evidence $E_{l}$ through the template $\mathcal{T}$. Now we formally define the transformation mapping from $\mathcal{N}_{l}$ to a computational graph:

- For each weighted ground fact $\left(V_{i}, e\right)$ occurring directly in $E_{l}$, there is a node $F_{\left(V_{i}, e\right)}$ in the computational graph, called a fact node.

- For each ground atom $h$ occurring in $\overline{\mathcal{N}} \backslash E_{l}$, there is a node $A_{h}$ in the computational graph, called an atom node.

- For every rule $c \leftarrow b_{1} \wedge \cdots \wedge b_{k} \in \mathcal{T}$ and every grounding substitution $c \theta=h \in \overline{\mathcal{N}}$, there is a node $G_{\left(c \leftarrow b_{1} \wedge \cdots \wedge b_{k}\right)}^{c \theta=h}$ in the computational graph, called an aggregation node.

- For every ground rule $\alpha_{i} \theta=\left(c \theta \leftarrow b_{1} \theta \wedge \cdots \wedge b_{k} \theta\right) \in \overline{\mathcal{N}}$, there is a node $R_{\left(c \theta \leftarrow b_{1} \theta \wedge \cdots \wedge b_{k} \theta\right)}$ in the computational graph, called a rule node.

An overview of the correspondence between the logical and the neural model, together with the used notation is reviewed in Table 1 .

The nodes of the computational graph that we defined above are then interconnected so as to follow the derivation of the logical facts by the immediate consequence operator starting from $E_{l}$, i.e. starting from the fact nodes $F_{\left(V_{i}, e\right)}$ which have no antecedent inputs in the computational graph and simply output their associated values $\operatorname{out}\left(F_{\left(V_{i}, e\right)}\right)=V_{i}$. The fact nodes are then connected into rule nodes $R_{\alpha \theta}$, particularly a node $F_{\left(V_{i}, e\right)}$ will be connected into every node $R_{\alpha \theta}=R_{\left(c \theta \leftarrow b_{1} \theta \wedge \cdots \wedge b_{k} \theta\right)}$ where

\footnotetext{
${ }^{15}$ Another option is backward-chaining of the rules back from the associated query atom $(q)$ through $\mathcal{T}$ into $E_{l}$. Note that this choice is purely technical and, following proper logical inference in both cases, does not affect the resulting logical (or neural) model.

${ }^{16}$ Otherwise it is automatically considered false (or having a default value) via CWA.
} 


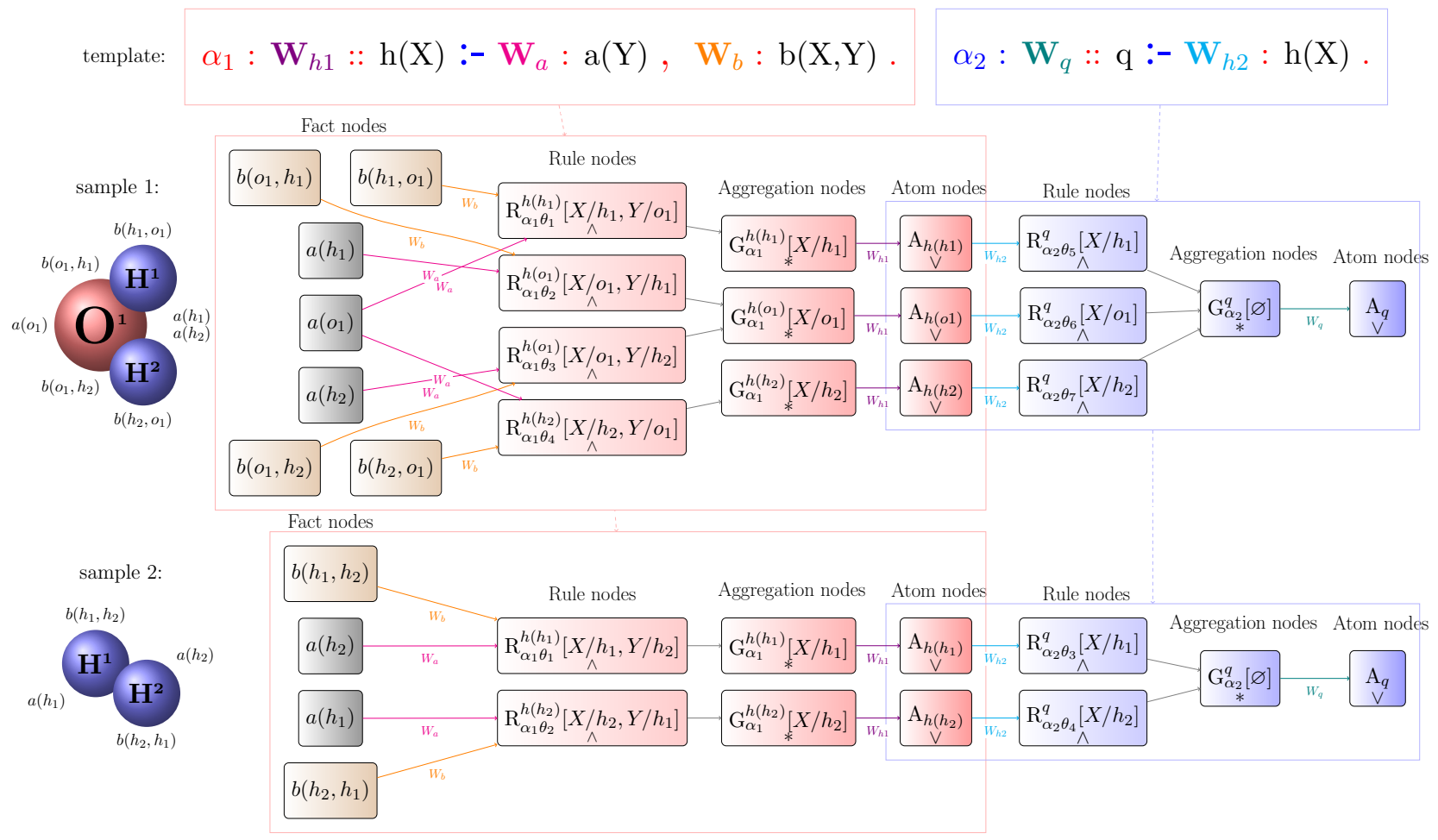

Figure 1: A simple LRNN template with 2 rules described in Example 1. Upon receiving 2 example molecules, 2 neural computation graphs get created, as prescribed by the semantics (Section 3.2).

$e=b_{i} \theta$ for some $i$. Having all the inputs, corresponding to the body literals of the associated ground rule, connected, the rule node will output a value calculated as

$$
\operatorname{out}\left(R_{\alpha \theta}\right)=g_{\wedge}\left(W_{1}^{\alpha} \cdot \operatorname{out}\left(F_{\left(V_{1}, b_{1} \theta\right)}\right), \ldots, W_{k}^{\alpha} \cdot \operatorname{out}\left(F_{\left(V_{i}, b_{k} \theta\right)}\right)\right) .
$$

The rule node's activation function $g_{\wedge}$ is up to user's choice. For scalar inputs, it can be for example set to mimic conjunction from Lukasiewicz logic, as in our previous work (Sourek et al., 2018). However, one can also choose to ignore the fuzzy-logical interpretation and use completely distributed semantics and activations utilized commonly in deep learning. In this case the computation follows the common (matrix) calculus by firstly aggregating the node's input values into its activation value

$$
\underset{(1 \times l)}{\operatorname{act}\left(R_{\alpha \theta}\right)}=\underset{(l \times n)}{W_{1}^{\alpha}} \cdot \underset{(1 \times n)}{\operatorname{out}\left(F_{1}\right)}+\cdots+\underset{(l \times m)}{W_{j}^{\alpha}} \cdot \underset{(1 \times m)}{\operatorname{out}\left(F_{k}\right)}
$$

followed by an element-wise application of any differentiable function, such as logistic sigmoid

$$
\underset{(1 \times l)}{\operatorname{out}\left(R_{\alpha \theta}\right)}=\underset{(1 \times l)}{\sigma\left(\operatorname{act}\left(R_{\alpha \theta}\right)\right)}=\sigma\left(\operatorname{act}(\operatorname{R\alpha } \theta)_{1}\right), \ldots, \sigma\left(\operatorname{act}(\operatorname{R\alpha } \theta)_{l}\right) .
$$

The rule nodes are then connected into aggregation nodes. Particularly, a rule node $R_{\left(c \theta \leftarrow b_{1} \theta \wedge \cdots \wedge b_{k} \theta\right)}$ is connected into the aggregation node $G_{\left(c \leftarrow b_{1} \wedge \cdots \wedge b_{k}\right)}^{c \theta=h}$ that corresponds to the same ground head literal $c \theta$. Having all the inputs, corresponding to different grounding substitutions $\theta_{i}$ of the rule $c \leftarrow$ $\left(b_{1} \wedge \cdots \wedge b_{k}\right)$ with the same ground head $h=c \theta_{1}=\cdots=c \theta_{q}$, connected, the aggregation node will output the value

$$
\operatorname{out}\left(G_{\alpha}^{c} \theta=h\right)=g_{*}\left(\operatorname{out}\left(R_{\alpha \theta_{1}}^{c \theta_{1}=h}\right), \ldots, \operatorname{out}\left(R_{\alpha \theta_{q}}^{c \theta_{q}=h}\right)\right) .
$$


where $g_{*}$ is some aggregation function, such as avg or max. The aggregation nodes effectively aggregate all the different ways by which a literal $h$ can be derived from a single rule $\alpha$. Their semantic intuitively corresponds to a certain quantification over free variables appearing solely in the rule's $\alpha$ body. The aggregation $g_{*}$ is then applied in each dimension of the input values as

$$
\begin{aligned}
& \left.\underset{1 \times l}{\operatorname{out}\left(G_{\alpha}^{h}\right)}=g_{*} \underset{1 \times l}{\operatorname{out}\left(R_{1}\right)}, \ldots, \underset{1 \times l}{\text { out }}\left(R_{q}\right)\right)=\left(g_{*}\left(\operatorname{out}\left(R_{1}\right)^{1}, \ldots, \text { out }\left(R_{q}\right)^{1}\right), \ldots,\right. \\
& \left.\ldots, g_{*}\left(\operatorname{out}\left(R_{1}\right)^{l}, \ldots, \text { out }\left(R_{q}\right)^{l}\right)\right) .
\end{aligned}
$$

The aggregation nodes are then connected into atom nodes. In particular, an aggregation node $G_{\alpha}^{h}$ will be connected into the atom node $A_{h}$ that is associated with the same atom $h$. The inputs of the atom node represent all the possible rules $\alpha_{i}$ through which the same atom $h$ can be derived. Having them all connected, $A_{h}$ will output the value

$$
\operatorname{out}\left(A_{h}\right)=g_{\vee}\left(W_{1}^{c} \cdot \operatorname{out}\left(G_{\alpha_{1}}^{h}\right), \ldots, W_{m}^{c} \cdot \operatorname{out}\left(G_{\alpha_{m}}^{h}\right)\right) .
$$

Apart from the choice of activation function $g_{\vee}$, the computation of the atom node's output follows exactly the same scheme as for the rule nodes.

Finally, the atom nodes are connected into rule nodes in exactly the same fashion as fact nodes, i.e. $A_{h}$ will be connected into every $R_{\left(c \theta \leftarrow b_{1} \theta \wedge \cdots \wedge b_{k} \theta\right)}$ where $h=b_{i} \theta$ for some $i$, and the whole process continues recursively.

Example 2 Let us follow up on the Example 1 by extending the described template with two example molecules of hydrogen and water. The template will then be used to dynamically unfold two computation graphs, one for each molecule, as depicted in Figure 1. Note that the computation graphs have different structures, following from the different Herbrand models derived from each molecule's facts, but share parameters in a scheme determined by the lifted structure of the joint template.

\section{Examples of Common Neural Architectures}

We demonstrate flexibility of the declarative LRNN templating, stemming from the abstraction power of Datalog, by encoding a variety of common neural architectures in very simple differentiable logic programs. For completeness, we start from simple neural models, where the advantages of templating are not so apparent, but continue to advanced deep learning architectures, where the expressiveness of relational templating stands out more clearly. Note that all templates in this paper are actual programs that can be run and trained with the LRNN interpreter.

\subsection{Feed-forward Neural Networks}

Multi-layer perceptrons (MLPs) form the most simple case where the weighted logic template is restricted to propositional clauses, and its single Herbrand model thus directly corresponds to a single neural model (Section 3.2). In this setting, the input example information can thus be encoded merely in the values of their associated tensors, which is the standard deep learning scenario. In the vector form, we can associate each example $E_{i}$ with a fact proposition $\left[\mathrm{v}_{1}^{\mathrm{i}}, \ldots, \mathrm{v}_{\mathrm{n}}^{\mathrm{i}}\right]::$ features $^{(0)}$, forming the input (0-th) node of the neural model. Each example is further associated with a query $\mathrm{v}_{\mathrm{q}}^{\mathrm{i}}:: q$.

In particular, an MLP with 3 layers, i.e. input layer ${ }^{(0)}, 1$ hidden layer $^{(1)}$, and output layer ${ }^{(2)}$, with the corresponding weight matrices $\left[\underset{\mathbf{m} \times \mathbf{n}}{\mathbf{W}}{ }^{(1)}, \underset{\mathbf{1} \times \mathbf{m}}{\mathbf{W}}{ }^{(2)}\right]$ can be directly modelled with the following rule

$$
{ }_{1 \times m}^{\mathbf{W}}(2):: q^{(2)}:-\underset{m \times n}{\mathbf{W}^{(1)}}: \text { features }{ }^{(0)} .
$$




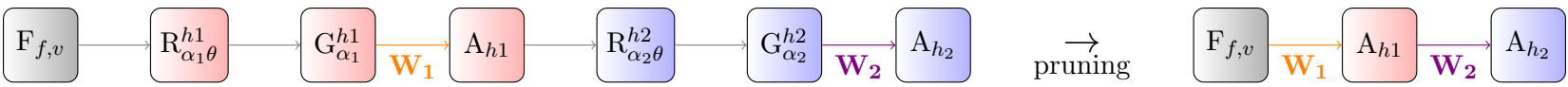

Figure 2: Demonstration of the pruning technique on a sample MLP model unfolded from a 2-rule template of $\alpha_{1}=\mathrm{W}_{1}:: h_{1}:-f$. and $\alpha_{2}=\mathbf{W}_{2}:: h_{2}:-h_{1}$.

Naturally, we can extend it to a deeper MLP by stacking more rules as

$$
\begin{aligned}
& 1 \underset{r \times m}{\mathbf{W}^{(2)}}:: \operatorname{hidden}^{(2)}:-\underset{\mathrm{m} \times \mathbf{n}}{\mathbf{W}^{(1)}} \text { : features }{ }^{(0)} \text {. } \\
& 2 \ldots \\
& 3 \underset{1 \times s}{\mathbf{W}^{(k)}}:: q^{(k)}:-\underset{s \times r}{\mathbf{W}^{(k-1)}}: \operatorname{hidden}^{(k-2)} .
\end{aligned}
$$

Once the template gets transformed into the corresponding neural model (Sec. 3.2), its computation graph will consist of a linear chain of nodes (Sec. 2.2.1) corresponding to standard fully-connected layers $1, \ldots, k$ with associated weight matrices $\left[W^{(1)}, W^{(2)} \ldots, W^{(k)}\right]$, and activation functions of user's choice.

Note that not all the weights need to be specified, and one can thus also write, e.g., either of

$$
1 \mathrm{~W}:: \mathrm{h}^{(2)}:-h^{(0)} \quad \mathrm{h}^{(2)}:-\mathrm{W}: h^{(0)} \text {. }
$$

While each of these rules still encodes in essence a 3-layer MLP, either only the hidden (right) or only the output (left) layer will carry learnable parameters, respectively. Moreover, following the exact semantics (Sec 3.2) for neural model creation, an aggregation node will be created on top of a rule node, representing the hidden layer. Since there is no need for aggregation in MLPs, i.e. only a single rule node ever gets created from each propositional rule, this introduces unnecessary operations in the graph. Since such nodes arguably do not improve learning of the model, we prune them out, as depicted in Figure 2. The technique is further described in more detail in Section A.1.1. Note that we assume application of pruning, where applicable, in the remaining examples described in this paper.

\subsubsection{Knowledge-based Artificial Neural Networks}

The direct correspondence between a propositional program and a neural network has been successfully exploited in a number of previous works, particularly the original Knowledge-Based Artificial Neural Networks (KBANN) (Towell et al., 1990), and LRNNs can be seen as a direct extension of KBANN into relational setting. To emulate the KBANN inference and learning, we simply fall back to scalar representation of features, e.g. 0::rain, 0.6::wet, 0.8::sunny, and consider a propositional template encoding some background domain knowledge, such as sprinkle :- wet, sunny. One then needs to choose a set of proper activation functions based on desired multi-valued logic semantics, e.g. the Lukasiewicz's fuzzy operators (Towell et al., 1990; Sourek et al., 2018). Note that, choosing proper fuzzy logic activations, this still covers standard logical inference as a special case with the use of binary fact values.

\subsection{Convolutional Neural Networks}

The CNNs can no longer be represented with a propositional template. To emulate the additional parts w.r.t. the MLPs, i.e. the convolutional filters and pooling (Sec. 2.2), we need to move to relational rules (Sec. 2.1). Note that there is a natural, close relationship between convolutions and 


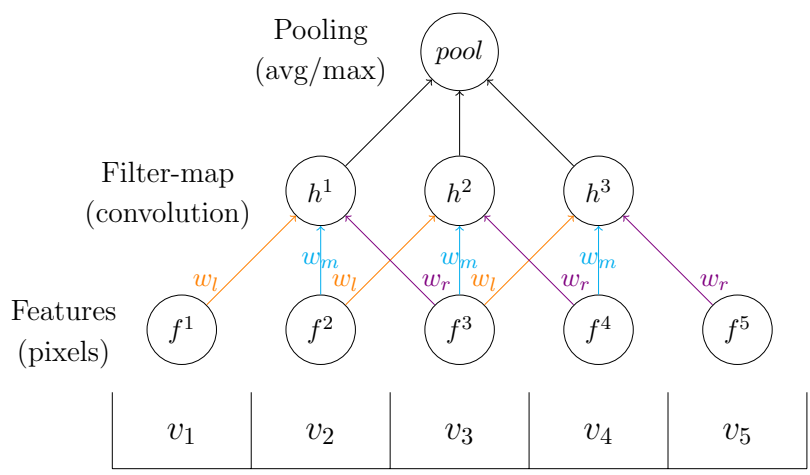

image $I$ as a vector of pixel values

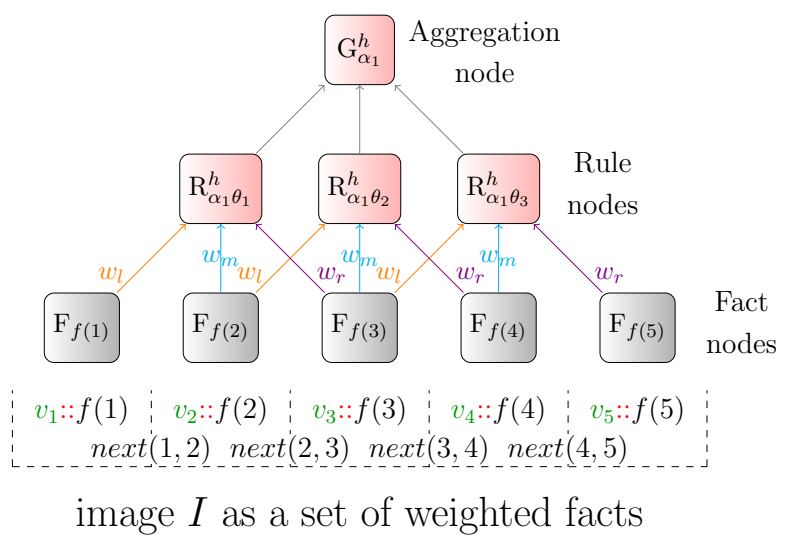

Figure 3: Left: core part of a standard CNN architecture with sparse layer composed of sequential applications of a convolutional filter (h), creating a feature-map layer, followed by a pooling operator. Right: the corresponding computation graph derived from a LRNN template.

relational rules (or relational patterns in general), where the point of both is to exploit symmetries in data. Moreover, the point of both the aggregation nodes and the pooling layers is to enforce certain transformation invariance. Let us demonstrate this relationship with the following example.

For clarity of presentation, consider a simplistic one-dimensional "image" consisting of 5 pixels $i=1, \ldots, 5$. While the regular grid structure of the image pixels is inherently assumed in CNN, we will need to encode it explicitly. Considering the 1-dimensional case, it is enough to define a linear ordering of the pixels such as next $(1,2), \ldots, n e x t(4,5)$. The (gray-scale) value $v_{i}$ of each pixel $i$ can then be encoded by a corresponding weighted fact $v_{i}: f(i)$. Next we encode a convolution filter of size $[1,3]$, i.e. vector which combines the values of each three ([left,middle,right $]$ ) consecutive pixels, and a (max/avg)-pooling layer that aggregates all the resulting values. This computation can be encoded using the following template

$1 \mathrm{~h}:-w_{l}: \mathrm{f}(\mathrm{A}), w_{m}: \mathrm{f}(\mathrm{B}), w_{r}: \mathrm{f}(\mathrm{C}), \operatorname{next}(\mathrm{A}, \mathrm{B}), \operatorname{next}(\mathrm{B}, \mathrm{C})$.

A visualization of the $\mathrm{CNN}$ and the corresponding computation graph derived from the logic model of the template presented with some example pixel values $\left[v_{1}, \ldots, v_{5}\right]$ is shown in Fig 3 .

While this does not seem like a convenient way to represent learning with CNNs from images, the important insight is that convolutions in neural networks correspond to weighted relational rules (patterns). The efficiency of normal CNN encoding is due to the inherent assumptions that are present in CNNs w.r.t. topology of their application domain, i.e. grids of pixel values, and similarly complete, ordered structures. While with LRNNs we need to state all these assumptions explicitly, it also means that we are not restricted to them - an advantage which will become clearer in the subsequent sections.

\subsection{Recursive and Recurrent Neural Networks}

A recursive network also exploits the principle of convolution, however the input is no longer a grid but a regular tree of an unknown structure. This prevents us from creating computation schemes customized to a specific structure, as in the CNNs. Instead, we need to resort to a general convolutional pattern that can be applied over any $k$-regular tree.

For that purpose, we again utilize the expressiveness of relational logic. Firstly, we encode the $k$-regular tree structure itself by providing a fact connecting each parent node in the tree to its child- 


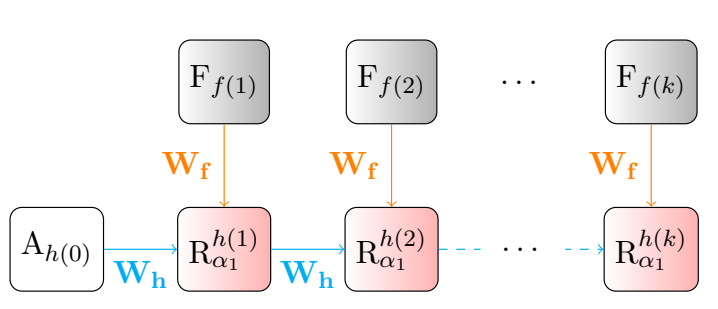

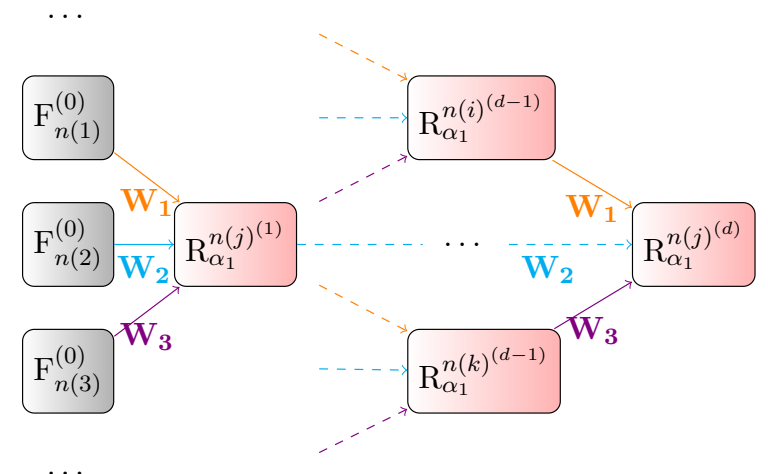

Figure 4: Simple recurrent (left) and recursive (right) neural structures encoded through LRNNs.

nodes, i.e. $\operatorname{parent}\left(\operatorname{node}_{j}^{i+1}, \operatorname{node}_{l}^{i}, \ldots, \operatorname{node}_{l+k}^{i}\right)$. Secondly, we associate all the leaf nodes in the tree with their embedding vectors $\left[\mathbf{v}_{1}^{i}, \ldots, \mathbf{v}_{\mathbf{n}}^{\mathbf{i}}\right]:: n\left(l e a f_{i}\right)$. Finally, a single relational rule can then be used to encode the recursive composition of representations in the, for instance 3-regular, tree as

$$
1 \mathrm{n}(\mathrm{P}):-\mathrm{W}_{1}: \mathrm{n}\left(\mathrm{C}_{1}\right), \mathrm{W}_{2}: \mathrm{n}\left(\mathrm{C}_{3}\right), \mathrm{W}_{3}: \mathrm{n}\left(\mathrm{C}_{3}\right) \text {, parent }\left(\mathrm{P}, \mathrm{C}_{1}, \mathrm{C}_{2}, \mathrm{C}_{3}\right) \text {. }
$$

which directly forms the whole learning template. Given a particular example tree, this rule translates to a computation graph recursively combining the children node representations $(n(C))$ into respective parent node representations, until the root node is reached. The root node representation $(n($ root $))$ could then be fed into a standard MLP rule (Sec. 4.1) to output the value for a given target query associated with the whole tree example.

A simple recurrent neural network unfolded over a linear (time) structure can then be modelled in a simpler manner, where only a single (vector) input is given at each step and a linear chain of hidden nodes $(h(X))$ replaces the prescribed tree hierarchy. Assuming encoding of the linear example structure with predicate next $(X, Y)$ as before, such a model can then be written as

$$
1 \mathrm{~h}(\mathrm{Y}):-\mathrm{W}_{\mathrm{f}}: \mathrm{f}(\mathrm{Y}), \mathrm{W}_{\mathrm{h}}: \mathrm{h}(\mathrm{X}), \operatorname{next}(\mathrm{X}, \mathrm{Y}) \text {. }
$$

The final hidden representation $(h(k))$ could then again be fed into a MLP for a whole sequence-level prediction. Neural architectures of both these templated models are displayed in Figure 4.

\section{Graph Neural Networks in LRNNs}

Graph (convolutional) Neural Networks (GNNs) (Sec. 2.3) can be seen as a generalization of the introduced neural architectures (Section 4) to arbitrary graphs, for which they combine the ideas of latent representation learning (Sec. 4.1), convolution (Sec. 4.2), and dynamic model structure (Sec. 4.3).

While modelling CNNs in the weighted logic formalism was somewhat cumbersome (because we had to explicitly represent the pixel grid), the encoding of GNNs is very straightforward. This is due to the underlying general graph representation with no additional assumptions of its structure, which 


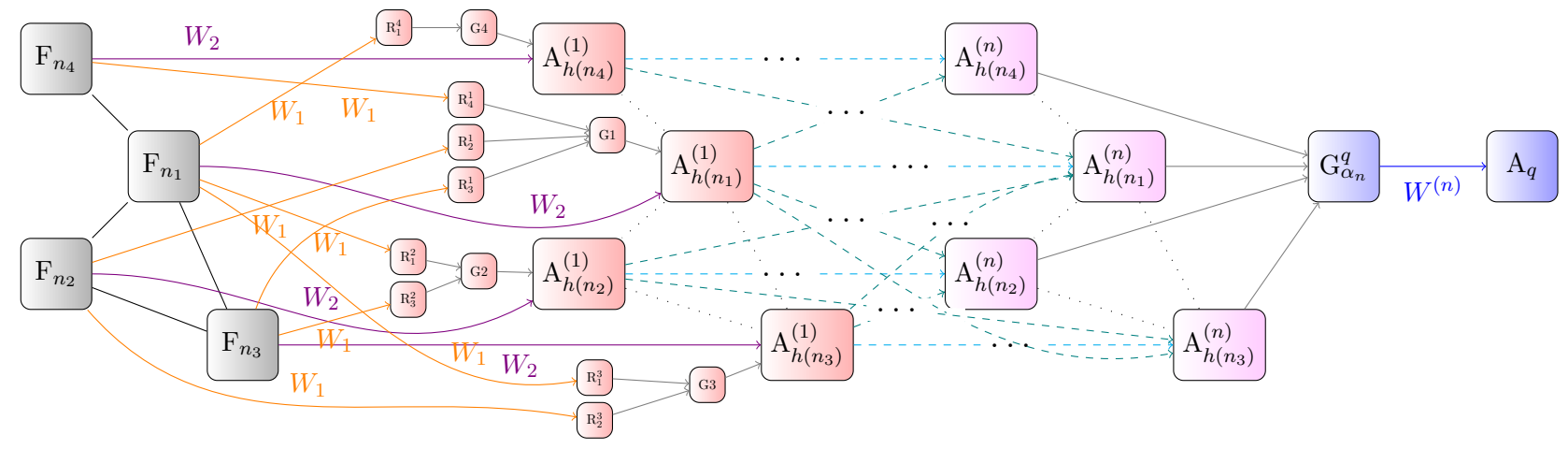

Figure 5: A computation graph of a sample (g-SAGE) GNN as encoded in LRNNs. Given an input graph of 4 (fact) nodes $\left(\mathrm{F}_{n_{1}} \ldots \mathrm{F}_{n_{4}}\right)$, neighbors of each node are firstly weighted and aggregated with rule and aggregation nodes, respectively (reduced in size in picture). The result is then combined with representation of the (central) node from the preceding layer, to form a new layer of 4 atom nodes, copying the structure of the input graph. After $n$ such layers, each with the same structure but different parameters, a global readout (aggregation) node aggregates all the node representations, passing to the final query (atom) node's transformation.

yields itself very naturally to relational logic. The computation of the layer $i$ update in GNNs can then be represented by a single rule as follows

$$
{ }_{1} \mathbf{W}^{(\mathrm{i})}:: \mathrm{h}^{(i)}(\mathrm{V}):-\mathrm{h}^{(i-1)}(\mathrm{U}), \operatorname{edge}(\mathrm{V}, \mathrm{U}) \text {. }
$$

where $e d g e / 2$ is the binary relation of the given input graphs. With the choice of activation functions as $g_{*}=$ avg,$g_{\wedge}=R e L U$, this simple rule can be already used to model the popular Graph Convolutional Neural Networks (GCN) (Kipf and Welling, 2016 ${ }^{17}$. The exact same rule (up to parameterization) is then used at each layer. For the final output query $(q)$ representing the whole graph we simply aggregate representations of all the nodes as

$$
{ }_{1} \mathbf{W}^{(\mathrm{d})}:: \quad \mathrm{q}:-\mathrm{h}^{(d-1)}(\mathrm{U})
$$

A noticeable shortcoming of GCNs is that the representation of the "central" node (V) itself is not used in the representation update. While this can be done by extending the graph (edge/2) with self-loops, a nove ${ }^{18}$ GNN model called GraphSAGE (g-SAGE) (Hamilton et al., 2017) was proposed to address this explicitly. To follow the architecture of g-SAGE, we thus split the template into 2 rules accordingly

$$
\begin{array}{ll}
{ }_{1} \mathrm{~h}^{(i)}(\mathrm{V}):-\mathrm{W}_{1}^{(\mathrm{i})}: \mathrm{h}^{(i-1)}(\mathrm{U}), \operatorname{edge}(\mathrm{V}, \mathrm{U}) \\
2 \mathrm{~h}^{(i)}(\mathrm{V}):-\mathrm{W}_{2}^{(\mathbf{i})}: \mathrm{h}^{(i-1)}(\mathrm{V})
\end{array}
$$

and choose $g_{\wedge}=R e L U, g_{*}=\max , g_{\vee}=i d e n t i t y$ for the very model (g-SAGE), the depiction of which can be seen in Figure 5 .

\footnotetext{
${ }^{17}$ where the authors also denoted the rule as convolution, since it forms a linear approximation of a localized spectral convolution (Kipf and Welling, 2016).

${ }^{18}$ Note that, differently from GCN with self-loops, the central node is parameterized differently from the neighbors.
} 
Another popular extension taken from neural architectures for image recognition are residual (skip) connections, where one effectively adds links to preceding layers at arbitrary depth (instead of just the preceding layer), i.e. we simply add one or more rules in the form

$$
{ }_{1} \mathrm{~W}_{\text {skip }}^{(\mathrm{i})}:: \mathrm{h}^{(i)}(\mathrm{V}):-\mathrm{h}^{(i-s k i p)}(\mathrm{V}) \text {. }
$$

This technique is also used in the Graph Isomorphism Network (GIN) (Xu et al., 2018a), which is a theoretically substantiated GNN based on the expressive power of the Weisfeiler-Lehman test (WL) (Weisfeiler, 2006). Firstly, the GIN model differs in that it adds residual connections from all the preceding layers to the final layer (which the authors refer to as "jumping knowledge" (Xu et al. 2018b)). Secondly, the particularity of GIN is to add a 2-layered MLP on top of each aggregation to harvest its universal approximation power. Particularly, update formula derived from the WLcorrespondence (Xu et al., 2018a) is

$$
h^{(i)}(v)=M L P^{(i)}\left(\left(1+\epsilon^{(i-1)}\right) \cdot h^{(i-1)}(v)+\sum_{u \in \mathcal{N}(v)} h^{(i-1)}(u)\right)
$$

where $M L P$ is the 2-layered MLP (Sec. 2.2.1). To accommodate the extra MLP layer, we thus extend the template as follows

$$
\begin{array}{ll}
1 & \operatorname{mlp}_{t m p}^{(i)}(\mathrm{V}):-\mathrm{h}(\mathrm{U})^{(i-1)}, \operatorname{edge}(\mathrm{V}, \mathrm{U}) \\
2 & \operatorname{mlp}_{t m p}^{(i)}(\mathrm{V}):-\left(1+\epsilon^{(\mathrm{i}-1)}\right): \mathrm{h}^{(i-1)}(\mathrm{V}) \\
{ }_{3} & \mathrm{~W}_{2}^{(\mathrm{i})}:: \mathrm{h}^{(i)}(\mathrm{V}):-\mathrm{W}_{1}^{(\mathrm{i})}: \operatorname{mlp}_{t m p}^{(i)}(\mathrm{V}) .
\end{array}
$$

Note that, considering that such a single rule actually already models a 2-layer ${ }^{19}$ MLP (as described in Sec. 4.1), a very similar computation can be carried out more simply with

$$
\begin{aligned}
& { }_{1} \mathbf{W}_{2 \mathbf{a}}^{(\mathrm{i})}:: \mathrm{h}^{(i)}(\mathrm{V}):-\mathrm{W}_{1 \mathrm{a}}^{(\mathrm{i})}: \mathrm{h}(\mathrm{U})^{(i-1)}, \operatorname{edge}(\mathrm{V}, \mathrm{U}) . \\
& { }_{2} \mathbf{W}_{\mathbf{2 b}}^{(\mathrm{i})}:: \mathrm{h}^{(i)}(\mathrm{V}):-\mathbf{W}_{1 \mathbf{b}}^{(\mathrm{i})}: \mathrm{h}^{(i-1)}(\mathrm{V}) .
\end{aligned}
$$

corresponding to a GIN version without the special $\left(1+\epsilon^{(i)}\right)$ coefficient, which the authors refer to as "GIN-0" (Xu et al. 2018a) and actually find performing better ${ }^{20}$, Finally they choose $g_{*}=$ sum as the function to aggregate the neighborhood representations. The authors proved the GIN model to belong to the most "powerful" class of GNN models, i.e. no other GNN model is more expressive than GIN, and demonstrated the GIN-0 model to provide state-of-the-art performance in various graph classification and completion tasks (Xu et al., 2018a).

\subsection{Extending GNNs}

While the GIN model presents the most "powerful" version of the basic GNN idea, there is a large number of ways in which the GNN approach can be extended. We discuss some of the direct, natural extensions in this subsection.

\footnotetext{
${ }^{19}$ or 3-layer, depending on inclusion of the input layer in the count.

${ }^{20}$ We note there is a slight difference, where GIN-0 firstly aggregates the neighbors and weights the result, while this template aggregates the neighbors after weighting. Nevertheless we note that GNN authors often switch this order themselves, for instance GraphSAGE in (Dwivedi et al. 2020) performs weighting before aggregation, while it is vice-versa in (Xu et al. 2018a).
} 


\subsubsection{Edge Representations}

Originally aimed at single-relation graphs, GNNs do not adequately utilize the information about the possibly different types of edges. While it is straightforward to associate edges with scalar weights in the adjacency matrix, instead of using just binary edge indicators (Kipf and Welling, 2016), extending to richer edge representations is not so direct, and has only been explored recently (Kipf et al., 2018. Gong and Cheng, 2019; Kim et al., 2019).

In the templating approach, addressing edges is very simple, since we do not operate directly on the graph but on the ground logical model, where each edge $\left(e d g e\left(n_{1}, n_{2}\right)\right)$ forms an atom in exactly the same way as the actual nodes $\left(\operatorname{node}\left(n_{1}\right)\right)$ in the graph itself (similarly to an extra transformation introduced in line-GNNs (Chen et al., 2017)). We can thus directly associate edges corresponding to different relations with arbitrary features $\left(\left[\mathbf{v}_{\mathbf{1}}, \ldots, \mathbf{v}_{\mathbf{n}}\right]::\right.$ edge $\left.\left(n_{1}, n_{2}\right)\right)$, learn their distributed representations, and predict their properties (or existence), just like GNNs do with the nodes. For basic learning with edge representations, there is no need to change anything in the previously introduced templates. However, one might want to associate extra transformations for edge and node representation learning (Gong and Cheng, 2019), in which case we would simply write

$$
{ }_{1} \mathbf{W}^{(\mathrm{i})}:: \mathrm{h}^{(i)}(\mathrm{V}):-\mathrm{h}(\mathrm{U})^{(i-1)}, \mathrm{W}_{\mathrm{e}}: \operatorname{edge}(\mathrm{V}, \mathrm{U}) \text {. }
$$

A large number of structured data then come in the form of multi-relational graphs, where the edges can take on different types. A straightforward extension is to learn a separate node representation of the nodes for each of the relations, e.g. as

$$
{ }_{1} \mathrm{~W}^{(\mathrm{i})}:: \mathrm{h}_{x}^{(i)}(\mathrm{V}):-\mathrm{h}_{x}(\mathrm{U})^{(i-1)}, \mathrm{W}_{\mathrm{e}}: \operatorname{edge}_{\text {type }=x}(\mathrm{~V}, \mathrm{U}) \text {. }
$$

and to choose from the different representations depending on context, such as in multi-sense word embeddings (Li and Jurafsky, 2015), or simply directly combine (Schlichtkrull et al., 2018) these representations in the template.

\subsubsection{Heterogeneous Graphs}

The majority of current GNNs then assume homogeneous graphs, and learning from heterogeneous graphs has just been marked as one of the future directions for GNNs (Wu et al., 2020$)$. In LRNNs, various heterogeneous graphs (Wang et al., 2019b) can be directly covered without any modification, since there is no restriction to the types of nodes and relations to be used in the same template (and so we do not have to e.g. split the graphs (Zhu et al. 2019) or perform any extra operation (Liu et al., 2018) for such a task). In the context of heterogeneous information networks, a similar "templating" idea has already become popular as defining "meta-paths" (Dong et al., 2017; Huang and Mamoulis, 2017), which can be directly covered by a single LRNN rule and, importantly, differentiated through.

We can further represent the relations as actual objects to be operated by logical means, by reifying them into logical constants as

$$
{ }_{1} \mathrm{~W}_{1}^{(\mathrm{i})}:: \mathrm{h}^{(i)}(\mathrm{V}):-\mathrm{h}(\mathrm{U})^{(i-1)}, \mathrm{h}(\mathrm{E})^{(i-1)}, \operatorname{edge}(\mathrm{V}, \mathrm{U}, \mathrm{E}) \text {. }
$$

where variable $E$ represents the edge object and $h(E)$ is its hidden representation. The learned embeddings of the nodes and relations can then be directly used for predicting triplets of (Object,Relation,Subject) in KBC, again with a simple template extension, e.g. for an MLP-based KBE (Dong et al., 2014), as

$$
1 \mathrm{~W}:: \operatorname{edge}(\mathrm{O}, \mathrm{R}, \mathrm{S}):-\mathrm{W}_{\mathrm{o}}: \mathrm{h}(\mathrm{O}), \mathrm{W}_{\mathrm{r}}: \mathrm{h}(\mathrm{R}), \mathrm{W}_{\mathrm{s}}: \mathrm{h}(\mathrm{S}) \text {. }
$$




\subsubsection{Hypergraphs}

Naturally, the GNN idea can be extended to hypergraphs, too, as was recently also proposed (Feng et al., 2019). While extending to hypergraphs from the adjacency matrix form used for simple graphs can be somewhat cumbersome, in the relational Datalog, hypergraphs are first-class citizens, so we can just directly write

$$
\begin{array}{ll}
1 & \mathrm{~W}_{1}^{(\mathrm{i})}:: \mathrm{h}^{(i)}\left(\mathrm{U}_{1}\right):-\mathrm{h}\left(\mathrm{U}_{1}\right)^{(i-1)}, \ldots, \mathrm{h}\left(\mathrm{U}_{n}\right)^{(i-1)} \text {, edge }\left(U_{1}, \ldots, U_{n}\right) . \\
2 & \ldots \\
3 & \mathrm{~W}_{1}^{(\mathrm{i})}:: \mathrm{h}^{(i)}\left(\mathrm{U}_{n}\right):-\mathrm{h}\left(\mathrm{U}_{1}\right)^{(i-1)}, \ldots, \mathrm{h}\left(\mathrm{U}_{n}\right)^{(i-1)} \text {, edge }\left(U_{1}, \ldots, U_{n}\right) .
\end{array}
$$

and possibly combine with all the other extensions.

There are many other simple ways in which GNNs can be extended towards higher expressiveness and there is a wide variety of emerging works in this area. While reaching beyond the standard, single adjacency matrix format, each of the novel extensions typically requires extra transformations (and libraries) to create their necessary intermediate representations (Chen et al., 2017; Dong et al., 2017). Many of these extensions are often deemed complex from the graph (GNN) point of view, but are rather trivial template modifications with LRNNs, as indicated in the preceding examples (and following in Sec. 5.2). This is due to the adopted declarative relational abstraction, as opposed to the procedural manipulations on ground graphs, defined often on a per basis. On the other hand we note that LRNNs currently cannot cover recent non-isotropic GNNs (Dwivedi et al., 2020) with computation constructs such as attention, gating, or LSTMs (Wu et al., 2020). While such construct could be included on an ad-hoc basis, they do not yield themselves naturally to the LRNN semantics.

\subsection{Beyond GNN architectures}

While we discussed possible ways for direct extensions of GNNs, there are more substantial alterations that break beyond the core principles of GNNs. One of them is the "message passing" idea, where the nodes are restricted to "communicate" with neighbors through the existing edges (WL label propagation (Weisfeiler, 2006)). Obviously, there is no such restriction in LRNNs, and we can design templates for arbitrary message passing schemes, corresponding to more complex and expressive convolutional filters. For instance, consider a simple extension beyond the immediate neighborhood aggregation by defining edges as weighted paths of length 2 (introduced as "soft edges" in (Sourek et al., 2018)):

$$
1 \text { W : : edge2(U,W) :- } \mathrm{W}_{1} \text { : edge }(\mathrm{U}, \mathrm{V}), \mathrm{W}_{2} \text { : edge }(\mathrm{V}, \mathrm{W}) \text {. }
$$

We can also easily compose the edges into small subgraph patterns of interest (also known as "graphlets" or "motifs" used in, e.g., social network analysis (Šourek et al., 2013)), such as triangles and other small cliques (alternatively conveniently representable by the hyper-edges (Sec. 5.1.3)), and operate on the level of these instead:

$$
{ }_{1} \mathrm{~W}:: \operatorname{node}(\mathrm{U}, \mathrm{V}, \mathrm{W}):-\mathrm{W}_{1} \text { : edge }(\mathrm{U}, \mathrm{V}), \mathrm{W}_{2} \text { : edge }(\mathrm{V}, \mathrm{W}), \mathrm{W}_{3} \text { : edge }(\mathrm{W}, \mathrm{U}) \text {. }
$$

Since both nodes and edges can be treated uniformly as logic atoms, we can easily alter the GNN idea to hierarchically propagate latent representations of the edges, too. In other words, each edge can aggregate representations of "adjacent" edges from previous layers:

$$
{ }_{1} \mathrm{~W}:: \mathrm{h}_{\text {edge }}^{(i)}(\mathrm{E}):-\mathrm{W}_{\mathrm{F}}: \mathrm{h}_{\text {edge }}^{(i-1)}(\mathrm{F}), \mathrm{W}_{\mathrm{U}, \mathrm{V}}: \operatorname{edge}(\mathrm{U}, \mathrm{V}, \mathrm{E}), \mathrm{W}_{\mathrm{V}, \mathrm{W}}: \operatorname{edge}(\mathrm{V}, \mathrm{W}, \mathrm{F}) .
$$


Naturally, this can be further combined with the standard learning of the latent node representations (as we do in experiments in Sec. 6).

Moreover, the messages do not have to spread homogeneously through the graph and a custom logic can drive the diffusion scheme. This can be, for instance, naturally put to work in the heterogeneous graph environments (Sec.5.1.2 with explicit types, which can then be used to control communication and representation learning of the nodes:

$$
1 \mathrm{~W}:: \mathrm{h}^{(i)}(\mathrm{X}):-\mathrm{h}^{(i-1)}(\mathrm{Y}) \text {, edge }(\mathrm{X}, \mathrm{Y}, \mathrm{E}) \text {, type }\left(\mathrm{E}, \operatorname{type}_{1}^{e}\right) \text {. }
$$

Besides being able to represented the types explicitly as objects (as opposed to the vector embeddings), we can actually induce new types, for instance into latent hierarchical categories (such as in (Šourek et al. 2016)):

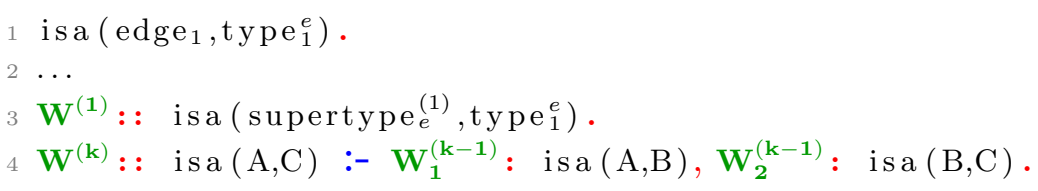

Importantly, there is no need to directly follow the input graph structure in each layer. We can completely abstract away from the graph representation in the subsequent layers and reason on the level of the newly invented, logically derived, entities, such as, e.g., the various graphlets, latent types, and their combinations:

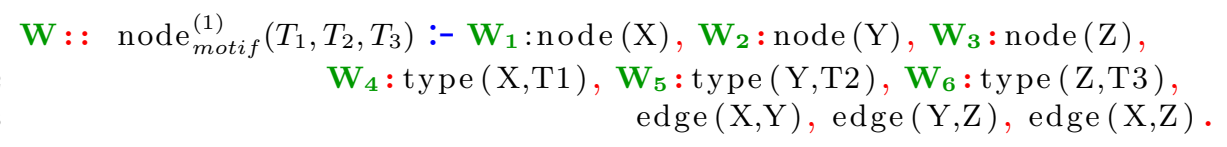

Finally, the models can be directly extended with external relational background knowledge. Note that such knowledge can be specified declaratively, with the same expressiveness as the templates themselves, since they are consequently simply merged together, for instance:

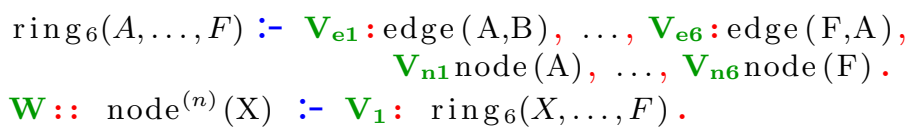

Note that this is very different from the standard GNNs, where one can only input ground information, in the form of numerical feature vectors along with the actual nodes (and possibly edges). Nevertheless this does not mean that LRNNs cannot work with numerical representations. On the contrary, besides the standard neural means, one can also directly interact with it by the logical means, e.g. by arithmetic predicates to define learnable numerical transformations (such as in (Sourek et al., 2018)) over some given (or learned) node similarities:

$$
1 \mathrm{~W}:: \operatorname{edge}_{\operatorname{sim}}\left(\mathrm{N}_{1}, \mathrm{~N}_{2}\right) \text { :- similar }\left(\mathrm{N}_{1}, \mathrm{~N}_{2}, \mathrm{~S}_{i m}\right), \mathrm{W}_{0.3}: \geq\left(\mathrm{S}_{i m}, 0.3\right) \text {. }
$$




\section{Experiments}

The preceding examples were meant to demonstrate high expressiveness and encoding efficiency of declarative LRNN templating. The main purpose of the experiments is to assess correctness and efficiency of the actual learning. For that purpose, we select GNNs as the most general and flexible of the commonly used neural architectures, since they encompass building blocks of all the other introduced architectures. Given the focus on GNNs, we compare against two most popular ${ }^{21}$ GNN frameworks of Pytorch Geometric (PyG) (Fey and Lenssen, 2019) and Deep Graph Library (DGL) (Wang et al. 2019a). Both these frameworks contain reference implementations of many popular GNN models, which makes them ideal for such a comparison. Note also that both these frameworks are highly contemporary and were specifically designed and optimized for creation and training of GNNs. For clarity of presentation, we restrict ourselves to a single task of structure property prediction, but perform experiments across a large number of datasets.

\subsection{Modern GNN frameworks}

While popular deep learning frameworks such as TensorFlow or Pytorch provide ways for efficient acceleration of standard neural architectures such as MLPs and CNNs, implementing GNNs is more challenging due to the irregular, dynamic, and sparse structure of the input graph data. Nevertheless, following the success of vectorization of the classic neural architectures, both PyG and DGL adopt the standard (sparse) tensor representation of all the data to leverage vectorized operations upon these. This includes the graphs themselves, which are then represented by their sparse adjacency matrices $G_{i}^{i}$. Further, each node index $i$ can be associated with a feature vector $\left(\left[f_{1}, \ldots, f_{j}\right]_{i}\right)$ through an additional matrix $F_{i}^{j}$ associated with each input graph.

Following the standard procedural differentiable programming paradigm, both frameworks then represent model computations explicitly through a predefined graph of tensor transformations applied directly to the input graph matrices, creating an updated feature tensor $F_{i}^{j^{(k)}}$ at each step $k$. The same tensor transformations are then applied to each input graph.

Both frameworks are based on similar ideas of message passing between the nodes (neighborhood aggregation) and its respective acceleration through optimized sparse tensor operations and batching (gather-scatter). DGL then seems to support a wider range of operations (and backends), with high-level optimizations directed towards larger scale data and models (and a larger overhead), while PyG utilizes more efficient low-level optimizations stemming from its tighter integration with Pytorch.

\subsection{Model and Training Correctness}

Firstly, we evaluate correctness of the templated GNN architectures via correspondence to their reference implementations in PyG and DGL. For this we select some of the most popular GNN models introduced in previous chapters, particularly the original GCN (Kipf and Welling, 2016), highly used GraphSAGE (g-SAGE) (Hamilton et al., 2017) and the "most powerful" GIN (Xu et al., 2018a) (particularly GIN-0). Each of the models comes with a slightly different aggregate-combine scheme and particular aggregation/activation functions (detailed in Sections 2.3 and 5). Moreover, we keep original GCN and g-SAGE as 2-layered models, while we adopt 5-layers for GIN (as proposed by the authors) ${ }^{22}$. We further use the same latent dimension $d=10$ for all the weights in all the models. Finally we set average-pooling operation, followed by a single linear layer, as the final graph-level readout for prediction in each of the models.

\footnotetext{
${ }^{21}$ with, as of date, PyG having $7.3 \mathrm{~K}$ stars and DGL having $4.7 \mathrm{~K}$ stars on Github, respectively.

${ }^{22}$ Obviously the number of layers could be increased/equalized for all of the models, however we keep them distinct to also accentuate their learning differences further.
} 


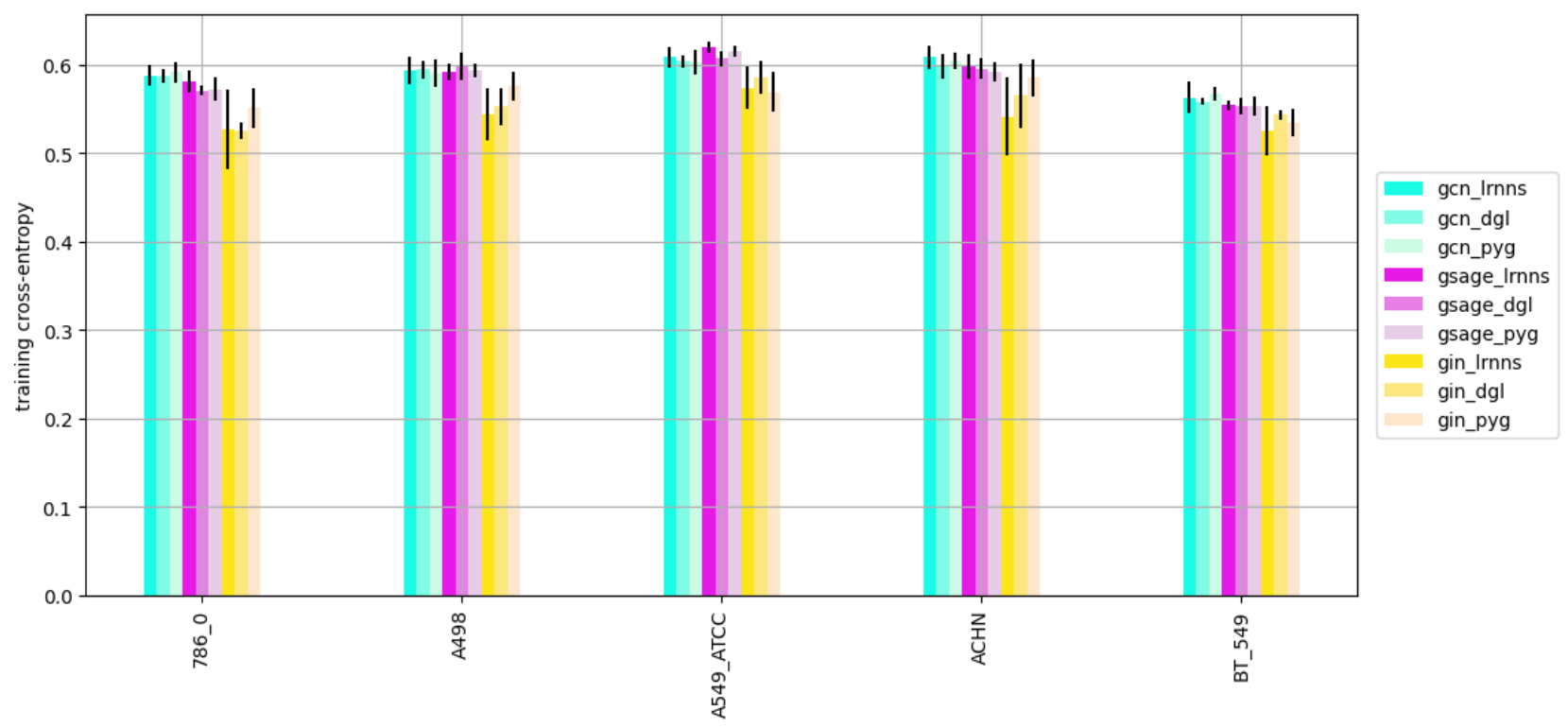

Figure 6: Alignment of training errors of the 3 models (GCN, g-SAGE, GIN), as implemented in the 3 different frameworks (LRNNs, DGL, PyG), over 5 datasets.

While the declarative templating takes a very different approach from the procedural GNN frameworks, for the specific case of GNN templates it is easy to align their computations, as they are mostly simple sequential applications of the (i) neighborhood aggregation, (ii) weighting, and (iii) non-linear activation, which can be covered altogether by a single rule (Section $5 \sqrt{23}^{2}$.

For the comparison, we choose the NCI (Ralaivola et al., 2005) molecular dataset:24, each containing thousands of molecules labeled by their ability to inhibit growth of different types of tumors. Note we only use the basic (Mol2 (Tripos, 2007)) types of atoms and bonds without extra chemical features. For visual clarity we present results only for the first 5 of the total 73 datasets in alphabetical order (while we note that the results are very similar over the whole set). We use the same 10-fold crossvalidation splits for all the models. We further use Glorot initialization scheme (Glorot and Bengio, 2010) where possible, and optimize using ADAM with a learning rate of $l r=1.5 e^{-5}$ (betas and epsilon kept the usual defaults) against binary crossentropy over 2000 epochae. Note that some other works propose a more radical training scheme with $l r=0.01$ and exponential decay by 0.5 every 50 epochae (Xu et al., 2018a), however we find GNN training in this setting highly unstable 25 and thus unsuitable for the alignment of the different implementations. We then report the actual training errors (as opposed to accuracy) as the most consistent evaluation metric for the alignment purpose in Figure 6. While it is very difficult to align the training perfectly due to the underlying stochasticity, we can see that the performances are tightly aligned within a margin of standard deviation calculated over the folds. The differences are generally highest for the most complex GIN model, which also exhibits most unstable performance over the folds. Importantly, the differences between LRNNs and the other frameworks is generally not greater than between PyG and DGL themselves, which both utilize the exact same PyTorch modules and operations.

\footnotetext{
${ }^{23}$ However for a precise correspondence, care must be taken to respect the same order of the (i-iii) operations, which often varies across different reports and implementations.

24 available at ftp://ftp.ics.uci.edu/pub/baldig/learning/nci/gi50/

${ }^{25}$ as is e.g. also visible in the respective Fig.4 reported in (Xu et al. $\left.2018 \mathrm{a}\right)$.
} 
Table 2: Training times per epocha across the different models and frameworks. Additionally, the startup model creation time (theorem proving) overhead of LRNNs is displayed.

\begin{tabular}{c|c|c|c|c} 
model/engine & LRNNs (s) & PyG (s) & DGL (s) & LRNNs startup (s) \\
\hline \hline GCN & $\mathbf{0 . 2 5} \pm \mathbf{0 . 0 1}$ & $3.24 \pm 0.02$ & $23.25 \pm 1.94$ & $35.2 \pm 1.3$ \\
\hline g-SAGE & $\mathbf{0 . 3 4} \pm \mathbf{0 . 0 1}$ & $3.83 \pm 0.04$ & $24.23 \pm 3.80$ & $35.4 \pm 1.8$ \\
\hline GIN & $\mathbf{1 . 4 1} \pm \mathbf{0 . 1 0}$ & $11.19 \pm 0.06$ & $52.04 \pm 0.41$ & $75.3 \pm 3.2$
\end{tabular}

\subsection{Computing Performance}

The main aim of the declarative LRNN framework is quick prototyping of models aiming to integrate deep and relational learning capabilities, for which it generally provides more expressive constructs than that of GNNs (Section 5) and does not contain any specific optimizations for computation over graph data. Additionally, it introduces a startup model compilation overhead as the particular models are not specified by the user but rather automatically induced by the theorem prover. Moreover, it implements the neural training in a rather direct (but flexible) fashion of actual traversal over each network (such as in Dynet (Neubig et al., 2017)), and does so without batching, efficient tensor multiplication or GPU support ${ }^{26}$. Nevertheless, we show that the increased expressiveness does not come at the cost of computation performance.

We evaluate the training times of a GCN over 10 folds of a single dataset (containing app. 3000 molecules) over the different models. We set Pytorch as the DGL backend (to match PyG), and train on $\mathrm{CPU}^{27}$ with a vanilla SGD (i.e. batch size=1) in all the frameworks. From the results in Table 2 , we see that LRNNs surprisingly train significantly faster than PyG, which in turn runs significantly faster than DGL. While the performance edge of PyG over DGL generally agrees with (Fey and Lenssen, 2019 ${ }^{28}$, the (app. 10x) edge of LRNN seems unexpected, even accounting for the startup theorem proving overhead for the model creation (giving PyG a head start of app. 10 epochae). We account the superior performance of LRNNs to the rather sparse, irregular, small, dynamic graphs for which the common vectorization techniques, repeatedly transforming the tensors there and back, often create more overhead than speedup, making it more efficient to traverse the actual spatial graph representations (Neubig et al., 2017). Additionally, LRNNs are implemented in Java, removing the Python overhead, and contain some generic novel computation compression (Sourek and Zelezny, 2020) techniques (providing about 3x speedup for the GNN templates).

Note we also prevented the frameworks from batching over several graphs, which they do by embedding the adjacency matrices into a block-diagonal matrix. While (mini) batching has been shown to deteriorate model generalization (Masters and Luschi, 2018; Wilson and Martinez, 2003), it still remains the main source of speedup in deep learning frameworks (Keskar et al., 2016), and is a highlighted feature of PyG, too. We show the additional PyG speedup gained by batching in Figure 7 . While batching truly boosts the PyG performance significantly, it still lacks behind the non-batched LRNN\&29. For illustration, we additionally include an inflated version of the GCN model by a $10 \mathrm{x}$ increase of all the tensor dimensionalities. In this setting we can finally observe a performance edge from mini-batching, due to vectorization and GPU ${ }^{30}$, over the non-batched LRNN: ${ }^{31}$.

\footnotetext{
${ }^{26}$ However it is possible to export the networks to be trained by any neural backend rather than the native Java engine.

${ }^{27}$ We evaluated the training on CPU as in this problem setting the python frameworks run slower on GPU (Figure 7 ).

${ }^{28}$ We note that we run both frameworks in default configurations, and there might be settings in DGL for which it does not lag behind PyG so rapidly. Note that for the small models of GCN and g-SAGE it is 10x slower, while for the bigger GIN model only 5x, which is in agreement with DGL's focus on large scale optimization.

${ }^{29}$ While LRNNs currently do not support batching natively, it can be emulated by outsourcing the training to Dynet.

${ }^{30}$ Also note that we used a non-high-end Ge-Force $940 \mathrm{~m}$, and the performance boost could thus be even more significant.

${ }^{31}$ On the other hand note that $\mathrm{dim}=100$ is considerably high. Most implementations we found were in range $\{8,16,32\}$ and we did not observe any test performance improvement beyond $\mathrm{dim}=5$ with the reported datasets and models.
} 


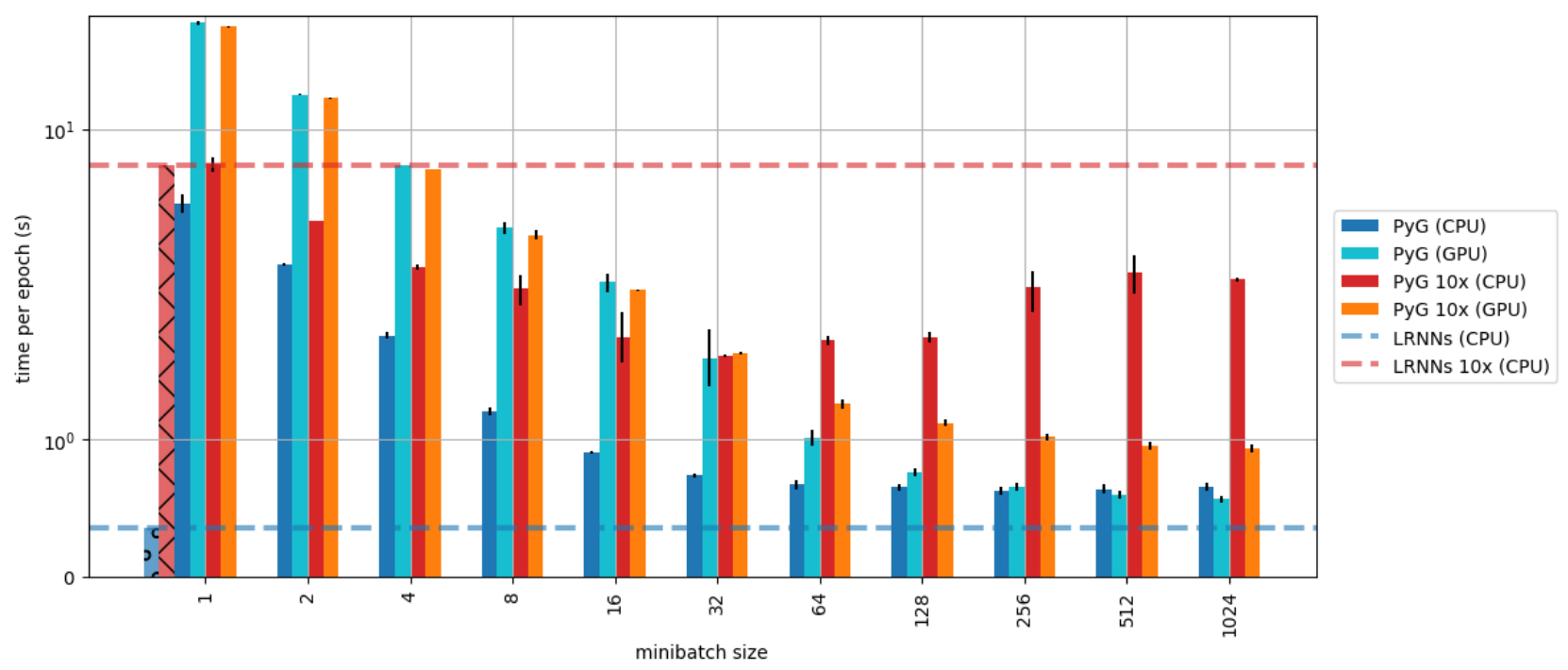

Figure 7: Improving the computing performance of PyG via mini-batching and model size blow-up. The actual GCN model $(10 \times 10$ parameter matrices $)$ and $10 x$ inflated version $(100 \times 100$ parameter matrices) as run on CPU and GPU. Compared to a non-batched (batch=1) LRNN run on CPU.

\subsection{Model Generalization}

Finally we evaluate learning performances of the different models. We select the discussed GNN models of GCN, g-SAGE and GIN (we keep only the PyG implementation for clarity), and we further include some example relational templates for demonstration. Particularly, we extend GIN with edge (bond) representations and associate all literals in all rules with learnable matrices (Sec. 5), denoted as "gin*". In a second template we add a layer of graphlets (motifs) of size 3, aggregating jointly representations of three neighboring nodes, on top of GIN, denoted as "graphlets". Lastly, we introduce latent bond learning (Sec. 5.2) into GIN, where bond (edge) representations are also aggregated into latent hierarchies, denoted as "latent_bonds". Note that we restrict these new relational templates to the same tensor dimensionalities and number of layers as GIN. For statistical soundness, we increase the number of datasets to the first 10 (alphabetically). We run all the models on the same 10-fold crossvalidation splits with a 80:10:10 (train:val:test) ratio, and keep the same, previously reported, training hyperparameters. We display the aggregated training errors in Figure 8, and the crossvalidated test errors, corresponding to the best validation errors in each split, in Figure 9 .

We can observe that the training performance follows intuition about capacity of each model, where the more complex models generally dominate the simpler ones. However, the increased capacity does not seem to consistently translate to better test performance (contrary to the intuition stated in $(\mathrm{Xu}$ et al. 2018a)). While we could certainly pick a subset of datasets to support the same hypothesis on test sets, we can generally see that none of the models actually performs consistently better than another, and even the simplest models (e.g. GCN) often outperform the "powerful" ones (GIN and its modifications), and the test performances are thus generally inconclusive ${ }^{32}$. While this is in contrast with the self-reported results accompanying the diverse GNN proposals on similar-sized graph datasets, it is in agreement with another (much larger) recent benchmark (Dwivedi et al., 2020).

Additionally, we include results of an old LRNN template reported in (Sourek et al., 2015), denoted as "lrnn(2015)". It was based on small graphlets of size 3, similarly to the "graphlets" template (and

\footnotetext{
${ }^{32}$ We note that the conclusion could be different for different types of datasets.
} 


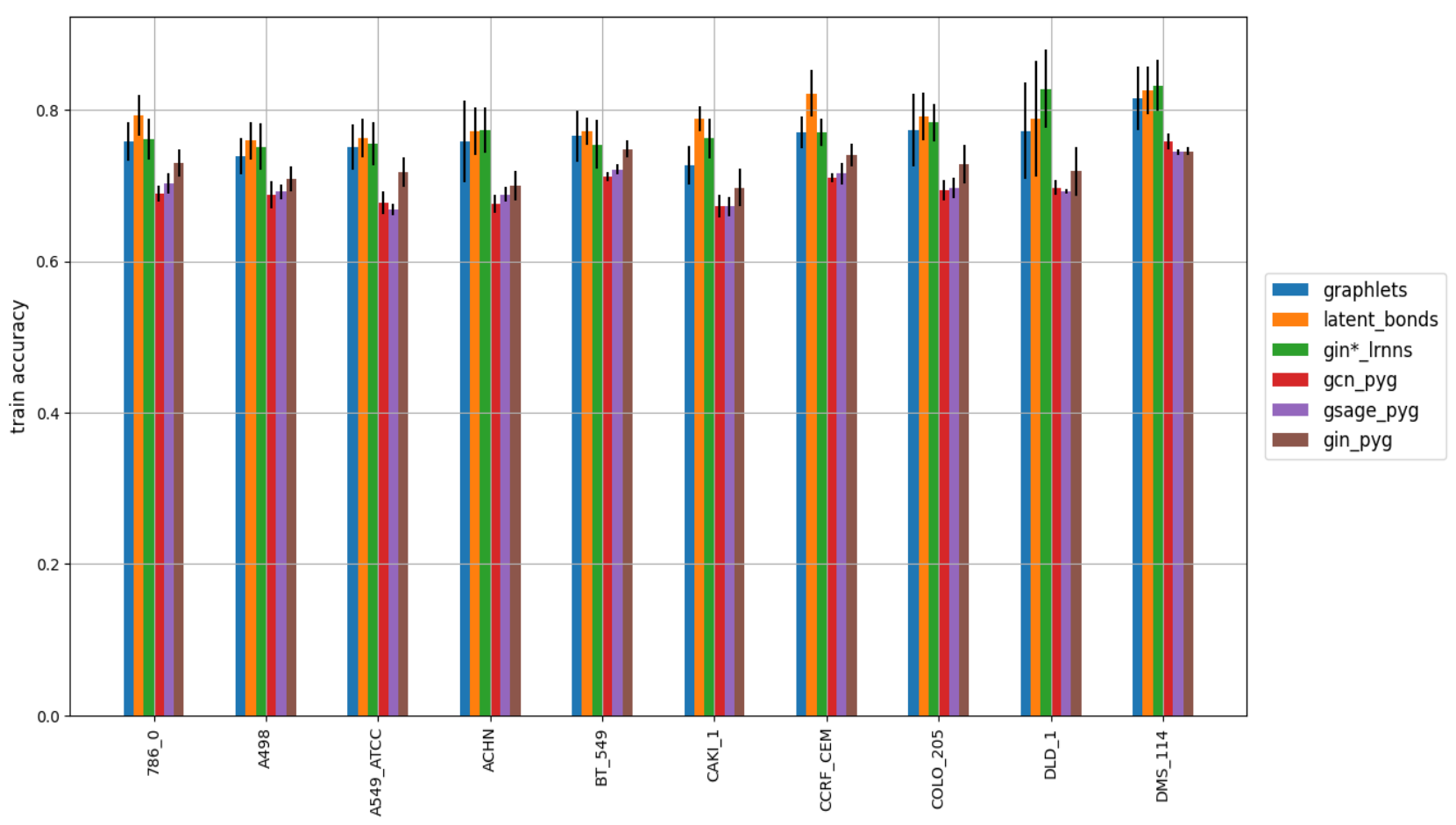

Figure 8: Comparison of train accuracies of selected models across 10 datasets.

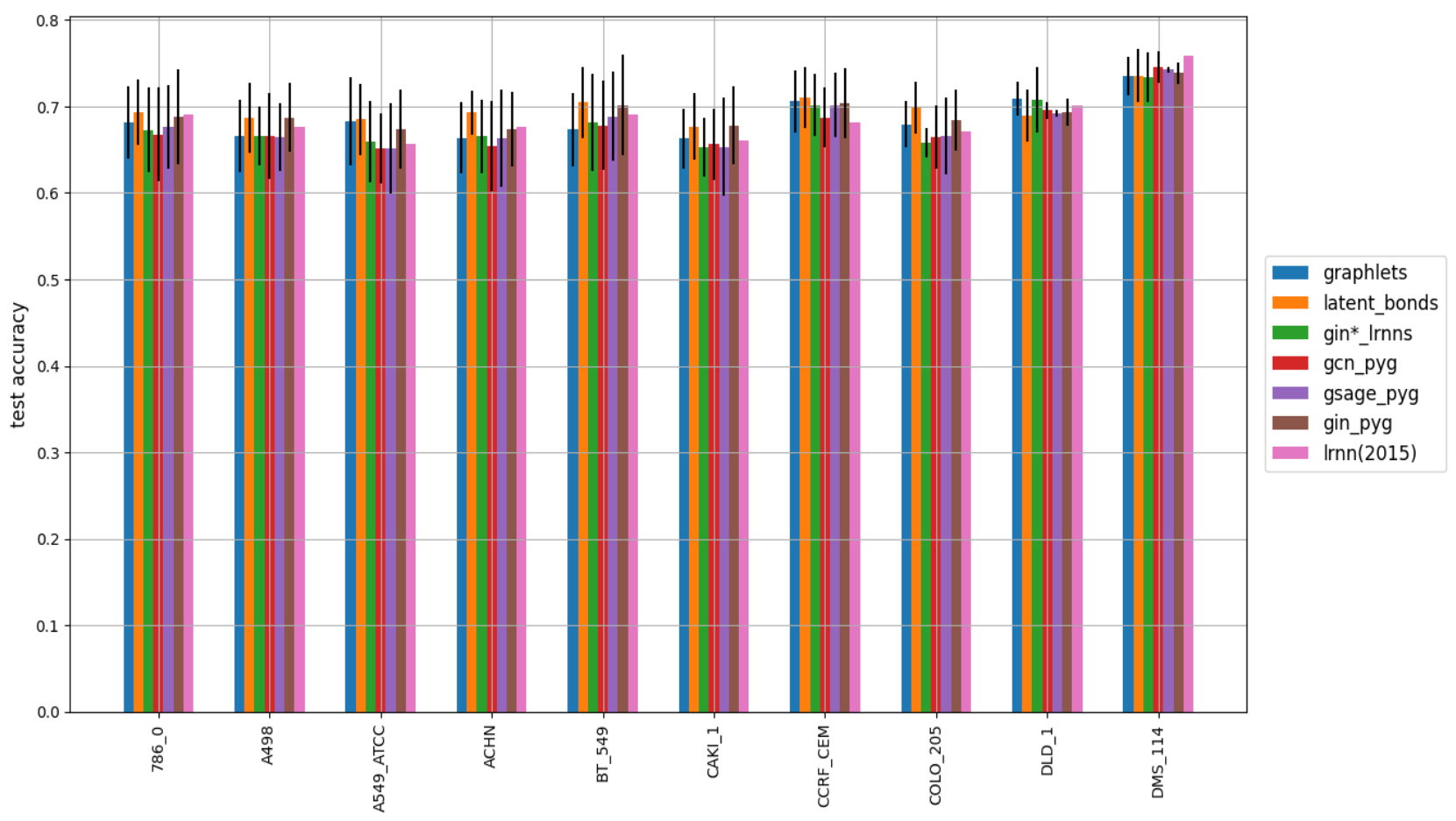

Figure 9: Comparison of test accuracies of selected models across 10 datasets. 
similarly to some other works (Tu et al. 2019; Sankar et al., 2017)), however it only contained a single layer of learnable parameters for the atom and bond representations. Note that we use results from the original paper (Sourek et al., 2015) experiments, which were run with different hyperparameters and splits. Nevertheless, we can see that it is again generally on par with performance of the more recent, deeper, and bigger GNN models.

\section{Related Works}

This work can be seen as a simple extension of the Lifted Relational Neural Networks (Šourek et al. 2015) language by increasing the amount of (tensor) parameterization. In turn LRNNs were inspired by lifted graphical models (Kimmig et al., 2015) such as Bayesian Logic Programs (Kersting and De Raedt, 2001) or Markov Logic Networks (Richardson and Domingos, 2006b), working in a probabilistic setting. From another view, LRNNs can also be seen as a direct extension of KBANN (Towell et al., 1990) into relational setting. The most closely related works naturally comprise of other differentiable programming languages with relational expressiveness (De Raedt et al., 2020)

There is a number of works targeting similar abilities by extending logic programming with numerical parameters. The most prominent framework in this category is the language of Problog (De Raedt et al. 2007), where the parameters and values further posses probabilistic interpretation. The extension of Deep-Problog (Manhaeve et al., 2018) then incorporates "neural predicates" into Problog programs. Since probabilistic logic programs can be commonly differentiated (Fadja et al., 2017) and trained as such, the gradients can be passed from the logic program to the neural modules and trained jointly. While this is somewhat similar to LRNNs, Deep-Problog introduces a clear separation line between the neural and logical parts of the program, which communicate merely through the gradient values (and so any gradient-based learner could be used instead). The logical part with relational expressiveness is thus completely oblivious of structure of the gradient-ingesting learner and vice versa, and it is thus impossible to model complex convolutional patterns (i.e. relational patterns in the neural part) as demonstrated in this paper. On the other hand LRNNs do not have probabilistic interpretation. Related is also a recent extension of kProblog (Orsini et al., 2017), proposing integration of algebraic expressions into logic programs towards more general tensor-algebraic and ML algorithms.

Neural Theorem Provers (NTPs) (Rocktäschel and Riedel, 2017) share very similar idea by the use of a relational logic template with a theorem prover to derive ground computation graphs, which are differentiable under certain semantics inspired by fuzzy logic. The use of parameterization differs between the frameworks, where NTPs are focused on learning embeddings of constants and LRNNs on embeddings of whole relational constructs34. Nevertheless NTPs represent all constants as embedding vectors, for which the theorem prover cannot perform standard unification, and NTPs thus resort to "soft-unification", effectively trying all possible constant combinations in the inference process. This prevents from using NTPs for explicit modelling of the exemplified convolutional neural architectures, and also severely limits NTP's scalability, where the latter has been partially addressed by some recent NTP extensions (Minervini et al., 2018; Weber et al., 2019). LRNNs are more flexible in this sense as one can use the parameterization to specify which parts of the program keep the logical structure and which parts should succumb themselves to the exhaustive numerical optimization (and to combine them arbitrarily), enabling to find a more fine-grained neural-symbolic trade-off.

\footnotetext{
${ }^{33}$ Note that common differentiable programming frameworks, such as PyTorch or TensorFlow, are effectively propositional. They provide sets of evaluation functions (modules), with predefined hooks for backward differentiation, that can be assembled by users into differentiable programs in a procedural fashion. In contrast, with relational programming, such programs are firstly automatically assembled from the declarative template (by a theorem prover), and only then evaluated and differentiated in the same fashion. Such approach could thus be understood as "meta-programming" (Visser, 2002, Hill and Gallagher 1998) from the perspective of the current procedural frameworks.

${ }^{34}$ Note that this includes learning embeddings of constants, too, as demonstrated in some of the example templates.
} 
Another line of work is focused on inducing Datalog programs with the help of numerical relaxation. While such a task has traditionally been addressed by the means of Inductive Logic Programming (ILP) (Muggleton and De Raedt, 1994), extending the rules with weights can help to relax the combinatorial search into a gradient descend optimization, while providing robustness to noise. An example of such an approach is $\delta$ ILP (Evans and Grefenstette, 2017). Similarly to LRNNs, Datalog programs are unfolded by chaining the rules, where the associated parameters are trained against given target to be solved by the program. The parameterization in these approaches is used differently as its purpose is to determine the right structure of the template. This is typically done by exhaustive enumeration from some restricted set of possible literal combinations (particularly 2 literals with arity at most 2 and no constants for $\delta \mathrm{ILP}$ ), where each combination is then associated with a weight to determine its appropriateness for the program via gradient descend. The differentiability is again based on replacing the logical connectives with fuzzy logic operators (particularly product t-norm). Similarly, programs in a restricted subset of Datalog are learned in a system called TensorLog (Cohen, 2016, Yang et al. 2017), which is a differentiable probabilistic database based on belief propagation (limited to tree-like factor graphs). Another recently proposed related system is called Difflog (Raghothaman et al., 2019), where the candidate rules are also exhaustively generated w.r.t. a more narrow language bias, thanks to which it seem to scale beyond previous systems. While we explicitly address only parameter learning in this paper, structure learning of the LRNN programs can also be done (Šourek et al., 2017).

Other class of approaches target full first order logic by providing mapping of all the logical constructs into numerical (tensor) spaces ("tensorization" (Garcez et al., 2019)). For instance, one can cast constants to vectors, functions terms to vector functions of the corresponding dimensionality, and similarly predicates to tensors of the corresponding arity-dimension (Rocktäschel et al., 2015, Diligenti et al., 2017). Again adopting a fuzzy logic interpretation of the logical connectives, the learning problem can then be cast as a constrained numerical optimization problem, including works such as Logic Tensor Networks (Serafini and Garcez, 2016) or LYRICS (Marra et al., 2019). While the distributed representation of the logical constructs is the subject of learning, in contrast with the discussed Datalog program structure learning approaches, the weight (strength) of each rule needs to be specified apriori - a limitation which was recently addressed in (Marra et al., 2020). Other recent works based on the idea of fully dissolving the logic into tensors, moving even further from the logical interpretation, include e.g. Neural Logic Machines (Dong et al., 2019). While these frameworks are theoretically more expressive than LRNNs (lacking the function terms and non-definite clauses), the whole logic interpretation is only approximate and completely dissolved in the tensor weights in these frameworks. Consequently, they lack the capability of precise relational logic inference chaining which we use to explicitly model the advanced convolutional neural structures, such as GNNs, in this paper.

Alternatively, LRNNs can be seen as an extension of GNNs, as discussed in this paper. From the graph-level perspective, the most similar idea to the introduced relational templating has become popular in the knowledge discovery community as "meta-paths" (Dong et al., 2017; Huang and Mamoulis, 2017) defined on the schema-level of a heterogeneous information network. A meta-path is simply a sequence of types, the concrete instantiations of which are then searched for in the ground graphs. Such ground sequences can then be used to define node similarities (Sun et al., 2011; Shang et al. 2016), random walks (Dong et al., 2017) as well as node embeddings (Shi et al., 2018; Fu et al., 2017). An extension from paths to small DAGs was then proposed as "meta-graph" (or "meta-structure") (Huang et al. 2016; Sun et al., 2018). Any meta-path or meta-graph can be understood as conjunctive a rule in a LRNN template. Naturally, we can stack multiple meta-graphs to create deep hierarchies and, importantly, differentiate them through to jointly learn all the parameters, and provide further extensions towards relational expressiveness exemplified in this paper. 


\section{Conclusions}

We introduced a differentiable declarative programming approach for specification of advanced relational neural architectures, based on the language of Lifted Relational Neural Networks (LRNNs) (Sourek et al., 2018). We demonstrated how simple parameterized logic programs, also called templates, can be efficiently used for declaration and learning of complex convolutional models, with a particular focus on Graph Neural Networks (GNNs). In contrast with the commonly used procedural (Python) frameworks, LRNNs abstract away the creation of the specific computation graphs, which are dynamically unfolded from the template by an underlying theorem prover. As a result, creating a diverse class of complex neural architectures reduces to rather trivial modifications of the templates, distilling only the high level idea of each architecture. We illustrated versatility of the approach on a number of examples, ranging gradually from simple neural models to complex GNNs, including very recent GNN models and their extensions. Finally we showed how the existing models can be easily extended to even higher relational expressiveness.

In the experiments, we then demonstrated correctness and computation efficiency by the means of comparison against modern deep learning frameworks. We showed that while LRNNs are designed with main focus on flexibility and abstraction, they do not suffer from computation inefficiencies for the simpler (GNN) models, as one might expect. On the contrary, we demonstrated that for a range of existing GNN models and their practical parameterizations, LRNNs actually outperform the existing frameworks optimized specifically for GNNs.

While there is a number of related works targeting the integration of deep and relational learning, to our best knowledge, capturing advanced convolutional neural architectures in an exact manner, as exemplified in this paper, would not be possible with the other approaches. The proposed relational upgrades can then be understood as proper extensions of the existing, arguably popular, GNN

models. However, we showed that generalization performance of various state-of-the-art GNN models is somewhat peculiar, as they actually performed with rather insignificant test error improvements, when measured uniformly over a large set of medium-sized, molecular structure-property prediction datasets, which is in agreement with another recent benchmark (Dwivedi et al., 2020). 


\section{A Appendix}

\section{A.1 Differences from LRNNs (Sourek et al., 2018)}

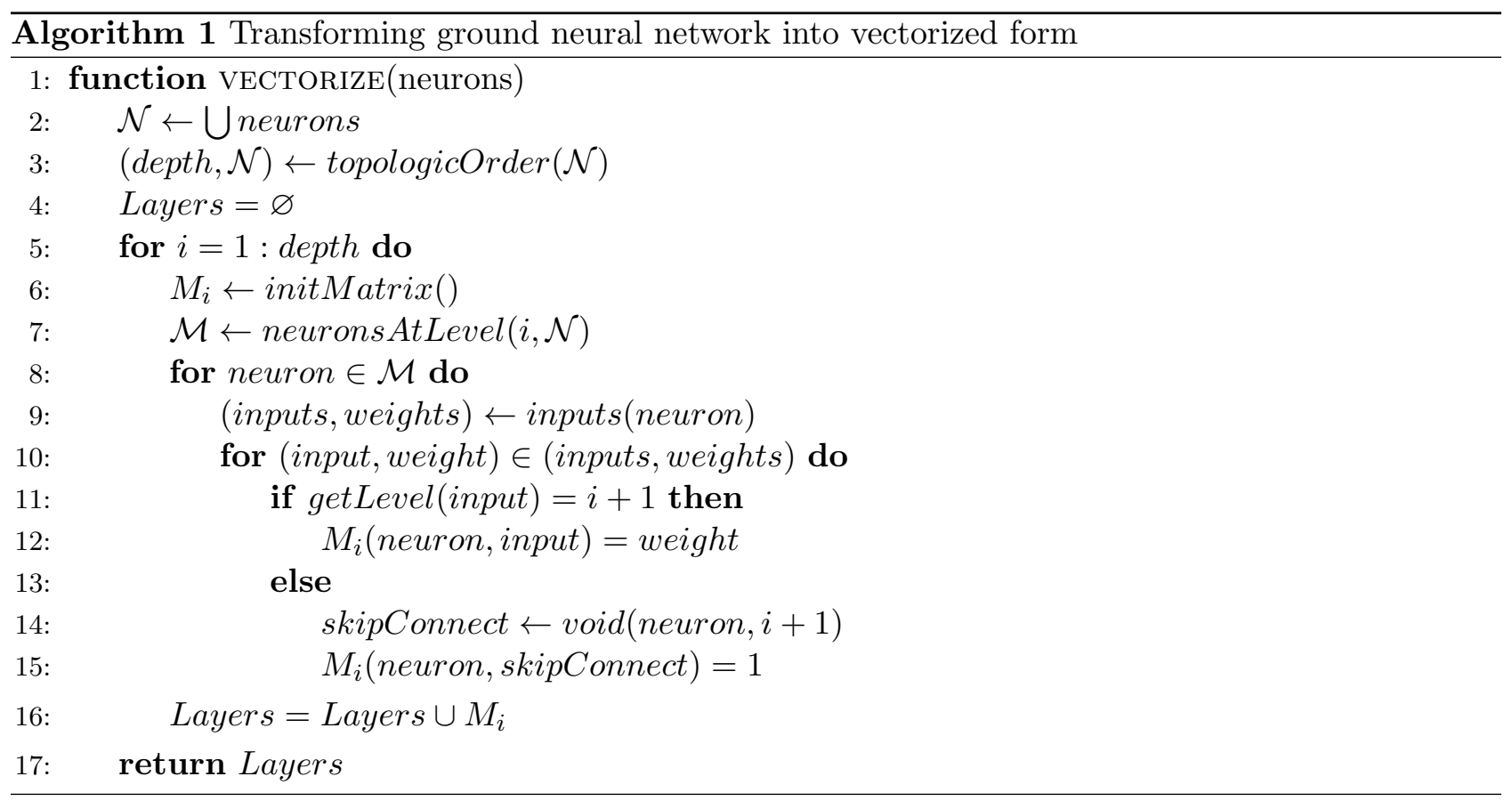

The framework introduced in this paper closely follows the original LRNNs (Sourek et al., 2018). In fact, the main semantic difference is "merely" in the parameterization of the rules, where one can now include the weights within the bodies (conjunctions), too, e.g.

$$
w_{1}^{(2)}:: \operatorname{node}_{1}^{(2)}(X) \leftarrow w_{1}^{(1)} \cdot \operatorname{node}_{1}^{(0)}(X) \wedge w_{2}^{(1)} \cdot \operatorname{node}_{2}^{(0)}(X)
$$

We note that this could be in essence emulated in the original LRNNs through the use of auxiliary predicates, such as in (Sourek et al., 2018), as follows

$$
\begin{aligned}
& w_{1}^{(2)}: \text { neuron }_{1}^{(2)}(X) \leftarrow \text { neuron }_{1}^{(1)}(X) \wedge \text { neuron }_{2}^{(1)}(X) \\
& w_{1}^{(1)}: \text { neuron }_{1}^{(1)}(X) \leftarrow \text { neuron }_{1}^{(0)}(X) \\
& w_{2}^{(1)}: \text { neuron }_{2}^{(1)}(X) \leftarrow \text { neuron }_{2}^{(0)}(X)
\end{aligned}
$$

which might be more appropriate in scenarios where the neurons correspond to actual logical concepts under fuzzy logic semantic:35, while the second representation is arguably more suitable to exploit the correspondence with standard deep learning architectures.

Another difference is that we now also allow tensor weights and values. While these could be modeled in LRNNs, too, for instance in the "soft-clustering" (embedding) construct (Šourek et al., 2015) used for atom representation learning:

$$
\begin{array}{lll}
w_{o_{1}}: g r_{1}(X) \leftarrow O(X) & \ldots & w_{h_{1}}: g r_{1}(X) \leftarrow H(X) \\
w_{o_{2}}: g r_{2}(X) \leftarrow O(X) & \ldots & w_{h_{2}}: g r_{2}(X) \leftarrow H(X)
\end{array}
$$

\footnotetext{
${ }^{35}$ Note that any model from the original LRNNs can still be directly encoded in the new formalism.
} 


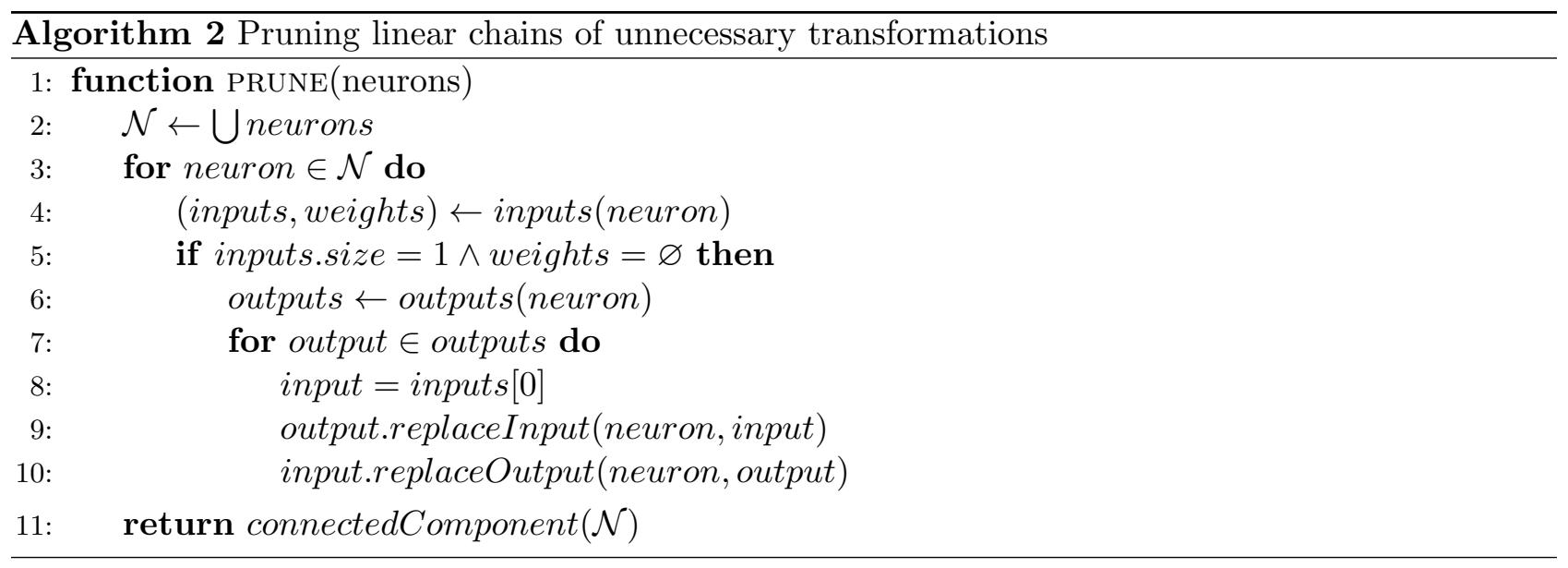

the tensor-valued weights offer an arguably more elegant representation of the same construct:

$$
\left[w_{o_{1}}, w_{o_{2}}\right]:: \operatorname{gr}(X) \leftarrow O(X) \quad \ldots \quad\left[w_{h_{1}}, w_{h_{2}}\right]:: \operatorname{gr}(X) \leftarrow H(X)
$$

In general, with the new representation we can merge scalar weights of individual neurons into tensors used by the nodes (prev. referred to as "neurons") in the computation graph. Note that we can process any ground LRNN network into this form, i.e. turn individual neurons into matrix layers, in a similar manner, as outlined in Algorithm 1.

Being heavily utilized in deep learning, such transformation can significantly speed up the training of the networks. However by reducing the number of rules, effectively merging together semantically equivalent rules which do not differ up to their (scalar) parameterization, we can also alleviate much of the complexity during model creation, i.e. calculation of the least Herbrand model, by avoiding repeated calculations. This results in a significant speedup during the model creation process.

\section{A.1.1 Network Pruning}

Following the computation graph creation procedure from Section 3.2 , we might end up with unnecessary trivial neural transformations through auxiliary predicates in cases, where the original rules have only a single literal in body and are unweighted. For mitigation, we can apply a straightforward procedure for detection and removal of linear chains of these trivial operations, as described in Algorithm 2. While such an operation arguably changes the inference and logical semantics of the original model, these structures do not contribute to learning capacity of the model and, on the contrary, cause gradient diminishing. This technique is thus particularly suited for improving training performance in correspondence with standard deep learning architectures. While this form of pruning can be theoretically performed directly in the template, it is easier to do as a post-processing step in the resulting neural networks.

Finally, the new LRNN framework ("NeuraLogic") presents a completely new implementation ${ }^{36}$ of the idea, with the whole functionality build from scratch, while aiming at flexibility and modularity.

\footnotetext{
${ }^{36}$ available at https://github.com/GustikS/NeuraLogic
} 


\section{References}

Bader S, Hitzler P (2005) Dimensions of Neural-symbolic Integration - A Structured Survey. arXiv preprint 0511042

Bancilhon F, Maier D, Sagiv Y, Ullman JD (1985) Magic sets and other strange ways to implement logic programs. In: Proceedings of the fifth ACM SIGACT-SIGMOD symposium on Principles of database systems, pp 1-15

Bistarelli S, Martinelli F, Santini F (2008) Weighted datalog and levels of trust. In: 2008 Third International Conference on Availability, Reliability and Security, IEEE, pp 1128-1134

Botta M, A G, Piola R (1997) Combining first order logic with connectionist learning. In: Proceedings of the 14th International Conference on Machine Learning

Bratko I (2001) Prolog programming for artificial intelligence. Pearson education

Bronstein MM, Bruna J, LeCun Y, Szlam A, Vandergheynst P (2017) Geometric deep learning: going beyond euclidean data. IEEE Signal Processing Magazine 34(4):18-42

Chen Z, Li X, Bruna J (2017) Supervised community detection with line graph neural networks. arXiv preprint arXiv:170508415

Cohen WW (2016) Tensorlog: A differentiable deductive database. arXiv preprint arXiv:160506523

Dash T, Srinivasan A, Vig L, Orhobor OI, King RD (2018) Large-scale assessment of deep relational machines. In: International Conference on Inductive Logic Programming, Springer, pp 22-37

De Raedt L, Kimmig A, Toivonen H (2007) Problog: A probabilistic prolog and its application in link discovery. In: IJCAI 2007, Proceedings of the 20th International Joint Conference on Artificial Intelligence, pp 2462-2467

De Raedt L, Kimmig A, Toivonen H (2007) Problog: A probabilistic prolog and its application in link discovery. In: IJCAI, Hyderabad, vol 7, pp 2462-2467

De Raedt L, Dumančić S, Manhaeve R, Marra G (2020) From statistical relational to neuro-symbolic artificial intelligence. arXiv preprint arXiv:200308316

Diligenti M, Gori M, Sacca C (2017) Semantic-based regularization for learning and inference. Artificial Intelligence 244:143-165

Ding L, Liya Ding (1995) Neural Prolog-the concepts, construction and mechanism. In: 1995 IEEE International Conference on Systems, Man and Cybernetics. Intelligent Systems for the 21st Century, IEEE, vol 4, pp 3603-3608, DOI 10.1109/ICSMC.1995.538347, URL http://ieeexplore.ieee. org/document/538347/

Dong H, Mao J, Lin T, Wang C, Li L, Zhou D (2019) Neural logic machines. arXiv preprint arXiv:190411694

Dong X, Gabrilovich E, Heitz G, Horn W, Lao N, Murphy K, Strohmann T, Sun S, Zhang W (2014) Knowledge vault: A web-scale approach to probabilistic knowledge fusion. In: Proceedings of the 20th ACM SIGKDD international conference on Knowledge discovery and data mining, pp 601-610 
Dong Y, Chawla NV, Swami A (2017) metapath2vec: Scalable representation learning for heterogeneous networks. In: Proceedings of the 23rd ACM SIGKDD international conference on knowledge discovery and data mining, pp 135-144

Dwivedi VP, Joshi CK, Laurent T, Bengio Y, Bresson X (2020) Benchmarking graph neural networks. arXiv preprint arXiv:200300982

Eisner J, Filardo NW (2010) Dyna: Extending datalog for modern ai. In: International Datalog 2.0 Workshop, Springer, pp 181-220

Evans R, Grefenstette E (2017) Learning Explanatory Rules from Noisy Data. To Appear in Journal of Artificial Intelligence Research Submitted 02, URL https://arxiv.org/pdf/1711.04574. pdfhttp://arxiv.org/abs/1711.04574, 1711.04574

Evans R, Saxton D, Amos D, Kohli P, Grefenstette E (2018) Can neural networks understand logical entailment? arXiv preprint arXiv:180208535

Fadja AN, Lamma E, Riguzzi F (2017) Deep probabilistic logic programming. In: PLP@ ILP, pp 3-14

Feng Y, You H, Zhang Z, Ji R, Gao Y (2019) Hypergraph neural networks. In: Proceedings of the AAAI Conference on Artificial Intelligence, vol 33, pp 3558-3565

Fey M, Lenssen JE (2019) Fast graph representation learning with pytorch geometric. arXiv preprint arXiv:190302428

Fu Ty, Lee WC, Lei Z (2017) Hin2vec: Explore meta-paths in heterogeneous information networks for representation learning. In: Proceedings of the 2017 ACM on Conference on Information and Knowledge Management, pp 1797-1806

Garcez Ad, Gori M, Lamb LC, Serafini L, Spranger M, Tran SN (2019) Neural-symbolic computing: An effective methodology for principled integration of machine learning and reasoning. arXiv preprint arXiv:190506088

Garcez ASA, Zaverucha G (1999) The connectionist inductive learning and logic programming system. Applied Intelligence 11(1):59-77

Gilmer J, Schoenholz SS, Riley PF, Vinyals O, Dahl GE (2017) Neural message passing for quantum chemistry. In: Proceedings of the 34th International Conference on Machine Learning-Volume 70, JMLR. org, pp 1263-1272

Glorot X, Bengio Y (2010) Understanding the difficulty of training deep feedforward neural networks. In: Proceedings of the thirteenth international conference on artificial intelligence and statistics, pp $249-256$

Gong L, Cheng Q (2019) Exploiting edge features for graph neural networks. In: Proceedings of the IEEE Conference on Computer Vision and Pattern Recognition, pp 9211-9219

Graves A, Wayne G, Danihelka I (2014) Neural Turing Machines. arXiv preprint URL http://arxiv. org/abs/1410.5401, 1410.5401

Graves A, Wayne G, Reynolds M, Harley T, Danihelka I, Grabska-Barwińska A, Colmenarejo SG, Grefenstette E, Ramalho T, Agapiou J, et al. (2016) Hybrid computing using a neural network with dynamic external memory. Nature 538(7626):471-476 
Hamilton W, Ying Z, Leskovec J (2017) Inductive representation learning on large graphs. In: Advances in neural information processing systems, pp 1024-1034

Hill P, Gallagher J (1998) Meta-programming in logic programming. Handbook of Logic in Artificial Intelligence and Logic Programming 5:421-497

Huang Z, Mamoulis N (2017) Heterogeneous information network embedding for meta path based proximity. arXiv preprint arXiv:170105291

Huang Z, Zheng Y, Cheng R, Sun Y, Mamoulis N, Li X (2016) Meta structure: Computing relevance in large heterogeneous information networks. In: Proceedings of the 22nd ACM SIGKDD International Conference on Knowledge Discovery and Data Mining, pp 1595-1604

Kadlec R, Bajgar O, Kleindienst J (2017) Knowledge base completion: Baselines strike back. arXiv preprint arXiv:170510744

Kazemi SM, Poole D (2018) Bridging Weighted Rules and Graph Random Walks for Statistical Relational Models. Frontiers in Robotics and AI 5:8, DOI 10.3389/frobt.2018.00008

Kersting K, De Raedt L (2001) Bayesian logic programs. arXiv preprint cs/0111058

Kersting K, De Raedt L (2001) Towards combining inductive logic programming with bayesian networks. In: Inductive Logic Programming, 11th International Conference, ILP 2001, Strasbourg, France, September 9-11, 2001, Proceedings, pp 118-131

Keskar NS, Mudigere D, Nocedal J, Smelyanskiy M, Tang PTP (2016) On large-batch training for deep learning: Generalization gap and sharp minima. arXiv preprint arXiv:160904836

Kim J, Kim T, Kim S, Yoo CD (2019) Edge-labeling graph neural network for few-shot learning. In: Proceedings of the IEEE Conference on Computer Vision and Pattern Recognition, pp 11-20

Kimmig A, Mihalkova L, Getoor L (2015) Lifted graphical models: a survey. Machine Learning 99(1):145

Kipf T, Fetaya E, Wang KC, Welling M, Zemel R (2018) Neural relational inference for interacting systems. arXiv preprint arXiv:180204687

Kipf TN, Welling M (2016) Semi-supervised classification with graph convolutional networks. arXiv preprint arXiv:160902907

Lamb L, Garcez A, Gori M, Prates M, Avelar P, Vardi M (2020) Graph neural networks meet neuralsymbolic computing: A survey and perspective. arXiv preprint arXiv:200300330

Li J, Jurafsky D (2015) Do multi-sense embeddings improve natural language understanding? arXiv preprint arXiv:150601070

Li Y, Tarlow D, Brockschmidt M, Zemel R (2015) Gated graph sequence neural networks. arXiv preprint arXiv:151105493

Lipton ZC, Berkowitz J, Elkan C (2015) A critical review of recurrent neural networks for sequence learning. arXiv preprint arXiv:150600019

Liu Z, Chen C, Yang X, Zhou J, Li X, Song L (2018) Heterogeneous graph neural networks for malicious account detection. In: Proceedings of the 27th ACM International Conference on Information and Knowledge Management, pp 2077-2085 
Manhaeve R, Dumancic S, Kimmig A, Demeester T, De Raedt L (2018) Deepproblog: Neural probabilistic logic programming. In: Advances in Neural Information Processing Systems, pp 3749-3759

Marcus G (2020) The next decade in ai: four steps towards robust artificial intelligence. arXiv preprint arXiv:200206177

Marra G, Giannini F, Diligenti M, Gori M (2019) Lyrics: a general interface layer to integrate ai and deep learning. arXiv preprint arXiv:190307534

Marra G, Diligenti M, Giannini F, Gori M, Maggini M (2020) Relational neural machines. arXiv preprint arXiv:200202193

Masters D, Luschi C (2018) Revisiting small batch training for deep neural networks. arXiv preprint arXiv:180407612

Minervini P, Bosnjak M, Rocktäschel T, Riedel S (2018) Towards Neural Theorem Proving at Scale. Tech. rep., URL https://arxiv.org/pdf/1807.08204.pdf, arXiv:1807.08204v1

Muggleton S, De Raedt L (1994) Inductive logic programming: Theory and methods. The Journal of Logic Programming 19

Neubig G, Dyer C, Goldberg Y, Matthews A, Ammar W, Anastasopoulos A, Ballesteros M, Chiang D, Clothiaux D, Cohn T, et al. (2017) Dynet: The dynamic neural network toolkit. arXiv preprint arXiv:170103980

Orsini F, Frasconi P, De Raedt L (2017) kproblog: an algebraic prolog for machine learning. Machine Learning 106(12):1933-1969

Palm R, Paquet U, Winther O (2018) Recurrent relational networks. In: Advances in Neural Information Processing Systems, pp 3368-3378

Raghothaman M, Si X, Heo K, Naik M (2019) Difflog: Learning datalog programs by continuous optimization

Ralaivola L, Swamidass SJ, Saigo H, Baldi P (2005) Graph kernels for chemical informatics. Neural Netw 18(8):1093-1110

Richardson M, Domingos P (2006a) Markov logic networks. Machine learning

Richardson M, Domingos P (2006b) Markov logic networks. Machine learning 62(1-2):107-136

Rocktäschel T, Riedel S (2017) End-to-end differentiable proving. In: Advances in Neural Information Processing Systems

Rocktäschel T, Singh S, Riedel S (2015) Injecting Logical Background Knowledge into Embeddings for Relation Extraction. Proceedings of the 2015 Conference of the North American Chapter of the Association for Computational Linguistics: Human Language Technologies pp 1119-1129, DOI 10.3115/v1/N15-1118, URL http://aclweb.org/anthology/N15-1118

Sankar A, Zhang X, Chang KCC (2017) Motif-based convolutional neural network on graphs. arXiv preprint arXiv:171105697

Scarselli F, Gori M, Tsoi AC, Hagenbuchner M, Monfardini G (2008) The graph neural network model. IEEE Transactions on Neural Networks 20(1):61-80 
Schlichtkrull M, Kipf TN, Bloem P, Van Den Berg R, Titov I, Welling M (2018) Modeling relational data with graph convolutional networks. In: European Semantic Web Conference, Springer, pp $593-607$

Serafini L, Garcez A (2016) Logic tensor networks: Deep learning and logical reasoning from data and knowledge. arXiv preprint arXiv:160604422

Serafini L, d'Avila Garcez AS (2016) Logic Tensor Networks: Deep Learning and Logical Reasoning from Data and Knowledge arXiv:1606.04422v1

Shang J, Qu M, Liu J, Kaplan LM, Han J, Peng J (2016) Meta-path guided embedding for similarity search in large-scale heterogeneous information networks. arXiv preprint arXiv:161009769

Shi C, Hu B, Zhao WX, Philip SY (2018) Heterogeneous information network embedding for recommendation. IEEE Transactions on Knowledge and Data Engineering 31(2):357-370

Smolensky P (1990) Tensor product variable binding and the representation of symbolic structures in connectionist systems. Artificial intelligence 46(1-2):159-216

Smullyan RM (1995) First-order logic. Courier Corporation

Socher R, Chen D, Manning CD, Ng A (2013a) Reasoning with neural tensor networks for knowledge base completion. In: Advances in neural information processing systems, pp 926-934

Socher R, Perelygin A, Wu JY, Chuang J, Manning CD, Ng AY, Potts C, et al. (2013b) Recursive deep models for semantic compositionality over a sentiment treebank. In: Proceedings of the conference on empirical methods in natural language processing (EMNLP), Citeseer, vol 1631, p 1642

Sourek G, Zelezny F (2020) Lossless compression of structured convolutional models via lifting. arXiv preprint

Šourek G, Kuzelka O, Zeleznỳ F (2013) Predicting top-k trends on twitter using graphlets and time features. ILP 2013 Late Breaking Papers p 52

Sourek G, Aschenbrenner V, Zelezny F, Kuzelka O (2015) Lifted relational neural networks. arXiv preprint arXiv: 150805128

Šourek G, Aschenbrenner V, Železny F, Kuželka O (2015) Lifted relational neural networks. In: Proceedings of the NIPS Workshop on Cognitive Computation: Integrating Neural and Symbolic Approaches co-located with the 29th Annual Conference on Neural Information Processing Systems (NIPS 2015).

Šourek G, Manandhar S, Železnỳ F, Schockaert S, Kuželka O (2016) Learning predictive categories using lifted relational neural networks. In: International Conference on Inductive Logic Programming, Springer, pp 108-119

Šourek G, Svatoš M, Železnỳ F, Schockaert S, Kuželka O (2017) Stacked structure learning for lifted relational neural networks. In: International Conference on Inductive Logic Programming, Springer, pp 140-151

Sourek G, Aschenbrenner V, Zelezny F, Schockaert S, Kuzelka O (2018) Lifted relational neural networks: Efficient learning of latent relational structures. Journal of Artificial Intelligence Research 62:69-100 
Sun L, He L, Huang Z, Cao B, Xia C, Wei X, Philip SY (2018) Joint embedding of meta-path and meta-graph for heterogeneous information networks. In: 2018 IEEE International Conference on Big Knowledge (ICBK), IEEE, pp 131-138

Sun Y, Han J, Yan X, Yu PS, Wu T (2011) Pathsim: Meta path-based top-k similarity search in heterogeneous information networks. Proceedings of the VLDB Endowment 4(11):992-1003

Towell GG, Shavlik JW, Noordewier MO (1990) Refinement of approximate domain theories by knowledge-based neural networks. In: Proceedings of the eighth National conference on Artificial intelligence, Boston, MA, pp 861-866

Tripos L (2007) Tripos mol2 file format. St Louis, MO: Tripos

Tu K, Li J, Towsley D, Braines D, Turner LD (2019) gl2vec: Learning feature representation using graphlets for directed networks. In: Proceedings of the 2019 IEEE/ACM International Conference on Advances in Social Networks Analysis and Mining, pp 216-221

Unman JD (1989) Principles of database and knowledge-base systems. Computer Science Press

Uwents W, Monfardini G, Blockeel H, Gori M, Scarselli F (2011) Neural networks for relational learning: an experimental comparison. Machine Learning 82(3):315-349, DOI 10.1007/ s10994-010-5196-5, URL https://doi.org/10.1007/s10994-010-5196-5

Van Emden MH, Kowalski RA (1976) The semantics of predicate logic as a programming language. Journal of the ACM (JACM) 23(4):733-742

Veličković P, Cucurull G, Casanova A, Romero A, Lio P, Bengio Y (2017) Graph attention networks. arXiv preprint arXiv:171010903

Visser E (2002) Meta-programming with concrete object syntax. In: International Conference on Generative Programming and Component Engineering, Springer, pp 299-315

Wang M, Yu L, Zheng D, Gan Q, Gai Y, Ye Z, Li M, Zhou J, Huang Q, Ma C, et al. (2019a) Deep graph library: Towards efficient and scalable deep learning on graphs. arXiv preprint arXiv:190901315

Wang X, Ji H, Shi C, Wang B, Ye Y, Cui P, Yu PS (2019b) Heterogeneous graph attention network. In: The World Wide Web Conference, pp 2022-2032

Weber L, Minervini P, Münchmeyer J, Leser U, Rocktäschel T (2019) Nlprolog: Reasoning with weak unification for question answering in natural language. arXiv preprint arXiv:190606187

Weisfeiler B (2006) On construction and identification of graphs, vol 558. Springer

Wilson DR, Martinez TR (2003) The general inefficiency of batch training for gradient descent learning. Neural networks 16(10):1429-1451

Wu Z, Pan S, Chen F, Long G, Zhang C, Yu PS (2019) A comprehensive survey on graph neural networks. arXiv preprint arXiv:190100596

Wu Z, Pan S, Chen F, Long G, Zhang C, Philip SY (2020) A comprehensive survey on graph neural networks. IEEE Transactions on Neural Networks and Learning Systems

Xu K, Hu W, Leskovec J, Jegelka S (2018a) How powerful are graph neural networks? arXiv preprint arXiv:181000826 
Xu K, Li C, Tian Y, Sonobe T, Kawarabayashi Ki, Jegelka S (2018b) Representation learning on graphs with jumping knowledge networks. arXiv preprint arXiv:180603536

Yang F, Yang Z, Cohen WW (2017) Differentiable learning of logical rules for knowledge base reasoning. In: Advances in Neural Information Processing Systems, pp 2319-2328

Zhou J, Cui G, Zhang Z, Yang C, Liu Z, Wang L, Li C, Sun M (2018) Graph neural networks: A review of methods and applications. arXiv preprint arXiv:181208434

Zhu S, Zhou C, Pan S, Zhu X, Wang B (2019) Relation structure-aware heterogeneous graph neural network. In: 2019 IEEE International Conference on Data Mining (ICDM), IEEE, pp 1534-1539 